\title{
FERMI DETECTION OF $\gamma$-RAY EMISSION FROM THE M2 SOFT X-RAY FLARE ON 2010 JUNE 12
}

M. Ackermani ${ }^{1}$, M. Ajello ${ }^{2}$, A. Allafort ${ }^{2}$, W. B. Atwood ${ }^{3}$, L. Baldini ${ }^{4}$, G. Barbiellini ${ }^{5,6}$, D. Bastieri ${ }^{7,8}$, K. BeChtol $^{2}$, R. Bellazzini ${ }^{4}$, P. N. Bhat ${ }^{9}$, R. D. Blandford ${ }^{2}$, E. Bonamente ${ }^{10,11}$, A. W. Borgland ${ }^{2}$, J. Bregeon ${ }^{4}$, M. S. BrigGs ${ }^{9}$,

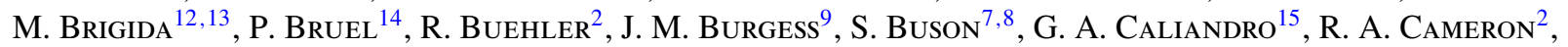
J. M. Casandian ${ }^{16}$, C. CeCchi ${ }^{10,11}$, E. Charles ${ }^{2}$, A. CheKhtman ${ }^{17,50}$ J. Chiang $^{2}$, S. Ciprini ${ }^{11,18}$, R. Claus ${ }^{2}$, J. Cohen-Tanugi ${ }^{19}$, V. Connaughton ${ }^{9}$, J. Conrad $20,21,51$, S. Cutini ${ }^{22}$, B. R. Dennis ${ }^{23}$, F. De Palma ${ }^{12,13}$, C. D. Dermer ${ }^{24}$, S. W. Digel ${ }^{2}$, E. do Couto e Silva ${ }^{2}$, P. S. Drell ${ }^{2}$, A. DrliCa-Wagner ${ }^{2}$, R. Dubois ${ }^{2}$, C. Favuzzi ${ }^{12,13}$, S. J. Fegan ${ }^{14}$, E. C. Ferrara ${ }^{23}$, P. Fortin ${ }^{14}$, Y. FuKazawa ${ }^{25}$, P. Fusco ${ }^{12,13}$, F. Gargano ${ }^{13}$, S. Germani ${ }^{10,11}$, N. Giglietto ${ }^{12,13}$, F. Giordano ${ }^{12,13}$, M. Giroletti ${ }^{26}$, T. Glanzman ${ }^{2}$, G. Godfrey ${ }^{2}$, L. Grillo ${ }^{2}$, J. E. Grove ${ }^{24}$, D. Gruber ${ }^{27}$, S. Guiriec ${ }^{9}$, D. Hadasch ${ }^{15}$, M. Hayashida ${ }^{2,28}$, E. Hays ${ }^{23}$, D. Horan ${ }^{14}$, G. IAfrate ${ }^{5,29}$, G. Jóhannesson ${ }^{30}$, A. S. Johnson ${ }^{2}$,

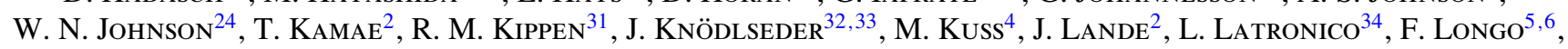
F. Loparco ${ }^{12,13}$, B. LotT ${ }^{35}$, M. N. Lovellette ${ }^{24}$, P. Lubrano ${ }^{10,11}$, M. N. Mazziotta ${ }^{13}$, J. E. McEnery ${ }^{23,36}$, C. Meegan $^{37}$, J. Mehault ${ }^{19}$, P. F. Michelson ${ }^{2}$, W. Mitthumsiri ${ }^{2}$, C. Monte $^{12,13}$, M. E. Monzani ${ }^{2}$, A. Morselli ${ }^{38}$, I. V. Moskalenko ${ }^{2}$, S. Murgia ${ }^{2}$, R. MurPhy ${ }^{24}$, M. Naumann-Godo ${ }^{16}$, E. Nuss ${ }^{19}$, T. Nymark ${ }^{21,39}$, M. Ohno ${ }^{40}$, T. Ohsugi ${ }^{41}$, A. Okumura ${ }^{2,40}$, N. Omodei ${ }^{2}$, E. Orlando ${ }^{2,27}$, W. S. Paciesas ${ }^{9}$, J. H. Panetta ${ }^{2}$, D. Parent ${ }^{42,50}$, M. Pesce-Rollins ${ }^{4}$, V. Petrosian ${ }^{2}$,

M. Pierbattista ${ }^{16}$, F. Piron ${ }^{19}$, G. Pivato ${ }^{8}$, H. Poon ${ }^{8}$, T. A. Porter ${ }^{2}$, R. Preece ${ }^{9}$, S. Rainò ${ }^{12,13}$, R. Rando ${ }^{7,8}$, M. Razzano ${ }^{4,3}$, S. Razzaque ${ }^{42,50}$, A. Reimer ${ }^{2,43}$, O. Reimer ${ }^{2,43}$, S. Ritz ${ }^{3}$, C. Sbarra 7 , R. A Schwartz ${ }^{23}$, C. Sgrò ${ }^{4}$, G. H. Share ${ }^{44,50,}$ E. J. Siskind ${ }^{45}$, P. Spinelli ${ }^{12,13}$, H. TAKahashi ${ }^{41}$, T. TANAKA ${ }^{2}$, Y. TANAKA ${ }^{40}$, J. B. ThaYeR ${ }^{2}$, L. Tibaldo ${ }^{7,8}$, M. Tinivella $^{4}$, A. K. Tolbert ${ }^{23}$, G. Tosti ${ }^{10,11}$, E. Troja ${ }^{23,52}$, Y. UchiYama ${ }^{2}$, T. L. Usher ${ }^{2}$, J. VAndenbroucke ${ }^{2}$, V. VAsileiou ${ }^{19}$, G. Vianello ${ }^{2,46,50}$, V. Vitale ${ }^{38,47}$, A. von Kienlin ${ }^{27}$, A. P. Waite ${ }^{2}$, C. Wilson-Hodge ${ }^{48}$, D. L. Wood ${ }^{49}$, K. S. WOOD ${ }^{24}$, AND Z. YANG ${ }^{20,21}$

${ }^{1}$ Deutsches Elektronen Synchrotron DESY, D-15738 Zeuthen, Germany

${ }^{2}$ W. W. Hansen Experimental Physics Laboratory, Kavli Institute for Particle Astrophysics and Cosmology, Department of Physics and SLAC National Accelerator Laboratory, Stanford University, Stanford, CA 94305, USA; nicola.omodei@ gmail.com

${ }^{3}$ Santa Cruz Institute for Particle Physics, Department of Physics and Department of Astronomy and Astrophysics, University of California at Santa Cruz, Santa Cruz, CA 95064, USA

${ }^{4}$ Istituto Nazionale di Fisica Nucleare, Sezione di Pisa, I-56127 Pisa, Italy

${ }^{5}$ Istituto Nazionale di Fisica Nucleare, Sezione di Trieste, I-34127 Trieste, Italy; francesco.longo@ trieste.infn.it ${ }^{6}$ Dipartimento di Fisica, Università di Trieste, I-34127 Trieste, Italy

${ }^{7}$ Istituto Nazionale di Fisica Nucleare, Sezione di Padova, I-35131 Padova, Italy

${ }^{8}$ Dipartimento di Fisica "G. Galilei," Università di Padova, I-35131 Padova, Italy

${ }^{9}$ Center for Space Plasma and Aeronomic Research (CSPAR), University of Alabama in Huntsville, Huntsville, AL 35899, USA; michael.briggs@nasa.gov

${ }^{10}$ Istituto Nazionale di Fisica Nucleare, Sezione di Perugia, I-06123 Perugia, Italy

${ }^{11}$ Dipartimento di Fisica, Università degli Studi di Perugia, I-06123 Perugia, Italy

12 Dipartimento di Fisica “M. Merlin” dell’Università e del Politecnico di Bari, I-70126 Bari, Italy

${ }^{13}$ Istituto Nazionale di Fisica Nucleare, Sezione di Bari, 70126 Bari, Italy

${ }^{14}$ Laboratoire Leprince-Ringuet, École polytechnique, CNRS/IN2P3, Palaiseau, France

${ }^{15}$ Institut de Ciències de l'Espai (IEEE-CSIC), Campus UAB, 08193 Barcelona, Spain

${ }^{16}$ Laboratoire AIM, CEA-IRFU/CNRS/Université Paris Diderot, Service d'Astrophysique, CEA Saclay, 91191 Gif sur Yvette, France

${ }^{17}$ Artep Inc., 2922 Excelsior Springs Court, Ellicott City, MD 21042, USA 18 ASI Science Data Center, I-00044 Frascati (Roma), Italy

${ }^{19}$ Laboratoire Univers et Particules de Montpellier, Université Montpellier 2, CNRS/IN2P3, Montpellier, France

${ }^{20}$ Department of Physics, Stockholm University, AlbaNova, SE-106 91 Stockholm, Sweden

${ }^{21}$ The Oskar Klein Centre for Cosmoparticle Physics, AlbaNova, SE-106 91 Stockholm, Sweden

${ }^{22}$ Agenzia Spaziale Italiana (ASI) Science Data Center, I-00044 Frascati (Roma), Italy

${ }^{23}$ NASA Goddard Space Flight Center, Greenbelt, MD 20771, USA

${ }^{24}$ Space Science Division, Naval Research Laboratory, Washington, DC 20375-5352, USA

${ }^{25}$ Department of Physical Sciences, Hiroshima University, Higashi-Hiroshima, Hiroshima 739-8526, Japan

${ }^{26}$ INAF Istituto di Radioastronomia, 40129 Bologna, Italy

${ }^{27}$ Max-Planck Institut für extraterrestrische Physik, 85748 Garching, Germany; dgruber@mpe.mpg.de

${ }^{28}$ Department of Astronomy, Graduate School of Science, Kyoto University, Sakyo-ku, Kyoto 606-8502, Japan

29 Osservatorio Astronomico di Trieste, Istituto Nazionale di Astrofisica, I-34143 Trieste, Italy

${ }^{30}$ Science Institute, University of Iceland, IS-107 Reykjavik, Iceland

${ }^{31}$ Los Alamos National Laboratory, Los Alamos, NM 87545, USA

${ }^{32}$ CNRS, IRAP, F-31028 Toulouse cedex 4, France

${ }^{33}$ GAHEC, Université de Toulouse, UPS-OMP, IRAP, Toulouse, France

${ }^{34}$ Istituto Nazionale di Fisica Nucleare, Sezioine di Torino, I-10125 Torino, Italy

${ }^{35}$ Université Bordeaux 1, CNRS/IN2p3, Centre d'Études Nucléaires de Bordeaux Gradignan, 33175 Gradignan, France

${ }^{36}$ Department of Physics and Department of Astronomy, University of Maryland, College Park, MD 20742, USA

${ }^{37}$ Universities Space Research Association (USRA), Columbia, MD 21044, USA

${ }^{38}$ Istituto Nazionale di Fisica Nucleare, Sezione di Roma "Tor Vergata," I-00133 Roma, Italy

${ }^{39}$ Department of Physics, Royal Institute of Technology (KTH), AlbaNova, SE-106 91 Stockholm, Sweden

${ }^{40}$ Institute of Space and Astronautical Science, JAXA, 3-1-1 Yoshinodai, Chuo-ku, Sagamihara, Kanagawa 252-5210, Japan

${ }^{41}$ Hiroshima Astrophysical Science Center, Hiroshima University, Higashi-Hiroshima, Hiroshima 739-8526, Japan

${ }^{42}$ Center for Earth Observing and Space Research, College of Science, George Mason University, Fairfax, VA 22030, USA

${ }^{43}$ Institut für Astro- und Teilchenphysik and Institut für Theoretische Physik, Leopold-Franzens-Universität Innsbruck, A-6020 Innsbruck, Austria

${ }^{44}$ Department of Astronomy, University of Maryland, College Park, MD 20742, USA; gerald.share@ nrl.navy.mil 


\author{
${ }^{45}$ NYCB Real-Time Computing Inc., Lattingtown, NY 11560-1025, USA \\ ${ }^{46}$ Consorzio Interuniversitario per la Fisica Spaziale (CIFS), I-10133 Torino, Italy \\ ${ }^{47}$ Dipartimento di Fisica, Università di Roma "Tor Vergata," I-00133 Roma, Italy \\ ${ }^{48}$ NASA Marshall Space Flight Center, Huntsville, AL 35812, USA \\ ${ }^{49}$ Praxis Inc., Alexandria, VA 22303, USA \\ Received 2011 October 15; accepted 2011 November 27; published 2012 January 12
}

\begin{abstract}
The Geostationary Operational Environmental Satellite (GOES) M2-class solar flare, SOL2010-06-12T00:57, was modest in many respects yet exhibited remarkable acceleration of energetic particles. The flare produced an $\sim 50 \mathrm{~s}$ impulsive burst of hard X- and $\gamma$-ray emission up to at least $400 \mathrm{MeV}$ observed by the Fermi Gamma-ray Burst Monitor and Large Area Telescope experiments. The remarkably similar hard X-ray and high-energy $\gamma$-ray time profiles suggest that most of the particles were accelerated to energies $\gtrsim 300 \mathrm{MeV}$ with a delay of $\sim 10 \mathrm{~s}$ from mildly relativistic electrons, but some reached these energies in as little as $\sim 3 \mathrm{~s}$. The $\gamma$-ray line fluence from this flare was about 10 times higher than that typically observed from this modest GOES class of X-ray flare. There is no evidence for time-extended $>100 \mathrm{MeV}$ emission as has been found for other flares with high-energy $\gamma$-rays.
\end{abstract}

Key words: acceleration of particles - Sun: flares - Sun: particle emission - Sun: X-rays, gamma rays

Online-only material: color figures

\section{INTRODUCTION}

The Sun is capable of accelerating electrons and ions to relativistic energies on time scales as short as a few seconds during solar flares. This conclusion has been reached based on observations of the X-rays, microwaves, $\gamma$-rays, and neutrons produced when the flare-accelerated particles interact in the solar atmosphere (Forrest \& Chupp 1983; Kane et al. 1986). The first reported observation of $\gamma$-rays with energies above $10 \mathrm{MeV}$ was made with the Solar Maximum Mission (SMM) spectrometer during the 1981 June 21 flare (Chupp et al. 1982). Most of the $\gamma$-ray emission occurred within an $\sim 70 \mathrm{~s}$ period and was followed minutes later by detection of high-energy neutrons at the spacecraft. Although it was clear from the neutron timing observations that protons were accelerated to energies in excess of $100 \mathrm{MeV}$, it was not possible from $\gamma$-ray spectroscopic studies to conclude that the protons reached the energies above $\sim 300 \mathrm{MeV}$ necessary to produce the characteristic spectrum from pion-decay radiation (Murphy et al. 1987).

Forrest et al. (1985, 1986) provided clear spectroscopic evidence for pion-decay emission during both the prompt and delayed emission phases of the 1982 June 3 flare. This emission in the second phase was confirmed by the presence of the $0.511-\mathrm{MeV}$ annihilation line from the decay of positively charged pions that had a time profile similar to the high-energy $\gamma$-rays (Share et al. 1983).

Since the era of those early measurements, improved spectrometers have detected the presence of pion-decay emission in several more flares and in two cases have observed $\gamma$-ray emission up to at least $1 \mathrm{GeV}$ (Kanbach et al. 1993; Akimov et al. 1996; Vilmer et al. 2003; Kuznetsov et al. 2011). Ryan (2000) and Chupp \& Ryan (2009) have reviewed these and other examples of high-energy $\gamma$-ray emission in flares. Most of these high-energy emissions have been observed over tens of minutes to hours leading to the designation of a class known as "Long Duration Gamma-Ray Flares." Several processes have been suggested to explain such particle acceleration to high energies in

\footnotetext{
$\overline{50}$ Resident at Naval Research Laboratory, Washington, DC 20375, USA.

${ }^{51}$ Royal Swedish Academy of Sciences Research Fellow, funded by a grant from the K. A. Wallenberg Foundation.

52 NASA Postdoctoral Program Fellow, USA.
}

the solar environment (Ellison \& Ramaty 1985; Petrosian et al. 1994; Park et al. 1997; Ryan 2000; Aschwanden 2004; Chupp \& Ryan 2009).

Typically such high-energy $\gamma$-ray emission has been associated with intense soft X-ray flares recorded by the Geostationary Operational Environmental Satellite (GOES) having peak powers exceeding $10^{-4} \mathrm{~W} \mathrm{~m}^{-2}$ (X class). It is of interest to determine whether less energetic solar flares have the capability of accelerating electrons to energies $\gtrsim 100 \mathrm{MeV}$ and protons to energies $\geqslant 300 \mathrm{MeV}$. Out of 24 flares observed at $\geqslant 10 \mathrm{MeV}$ energies by the $\gamma$-ray spectrometer on SMM, only 3 had GOES classifications of M5 or less (Vestrand et al. 1999). Similarly, most flares emitting $\gamma$-ray lines have been associated with intense soft $\mathrm{X}$-ray emission: out of $65 \gamma$-ray line flares observed by $S M M$, only 3 have been associated with flares having a GOES classification of M5 or less. Before detection of this flare, the Ramaty High Energy Solar Spectroscopic Imager (RHESSI) observed only one nuclear-line flare out of 20 below the M5 class (Shih et al. 2009) and that was characterized as C9.7. The smallest GOES-class flare for which detection of nuclear $\gamma$-rays has been claimed was the $\mathrm{C} 7$ flare observed by the COMPTEL Compton telescope on the Compton Gamma-Ray Observatory (CGRO; Young et al. 2001).

With the launch of the Fermi mission in 2008, it is now possible to make the high-sensitivity measurements necessary to detect $\geqslant 30 \mathrm{MeV} \gamma$-rays in the weakest flares. Fermi is comprised of two instruments: the Gamma-ray Burst Monitor (GBM; Meegan et al. 2009) sensitive from $\sim 8 \mathrm{keV}$ up to $40 \mathrm{MeV}$ and covering the energy band of nuclear $\gamma$-ray line emission; and the Large Area Telescope (LAT; Atwood et al. 2009) operating from $20 \mathrm{MeV}$ to more than $300 \mathrm{GeV}$ and covering most of the pion-decay emission energy range. In its normal sky survey mode the LAT observes the Sun for only $\sim 35$ minutes every $3 \mathrm{hr}$. It was therefore fortunate to observe the GOES M2class flare on 2010 June 12 (SOL2010-06-12T00:57). This flare produced nuclear line emission about an order of magnitude higher than typical for this magnitude soft X-ray flare. Although the flare lasted only about 2 minutes, it appears to have accelerated electrons and/or protons to energies $\geqslant 300 \mathrm{MeV}$ based on detection of $\gamma$-rays with energies $>100 \mathrm{MeV}$. One of the key features of this observation is that the high-energy 
emission is delayed by $\sim 5-10$ s relative to the $500-1000 \mathrm{keV}$ bremsstrahlung. This suggests that the acceleration of hundreds of keV electrons and hundreds of $\mathrm{MeV}$ electrons and/or protons in the chromosphere took place within $10 \mathrm{~s}$ of each other. A weak solar energetic particle (SEP) event observed by the GOES followed this flare.

In the next two sections, we discuss the capabilities of the GBM and LAT for detecting $\gamma$-rays from solar flares. These are followed by Section 4 describing the observations including spectroscopic studies in the nuclear energy range with the GBM and in the high-energy domain with the LAT. As many highenergy $\gamma$-ray flares previously observed exhibited an extended emission phase, we then discuss a search for such emission in this flare in the next section. In the final section, Section 5, we summarize our conclusions and discuss the results.

\section{GBM CAPABILITY FOR FLARE OBSERVATIONS}

The GBM was designed to observe gamma-ray bursts but has useful capabilities for other sources such as SGRs (soft $\gamma$-ray repeaters), pulsars, X-ray binaries using the Earth occultation technique, Terrestrial Gamma-ray Flashes (TGFs), and solar flares (Meegan et al. 2009). The GBM is comprised of 12 sodium iodide (NaI) detectors measuring the energy range from $\sim 8 \mathrm{keV}$ to $1 \mathrm{MeV}$, and 2 bismuth germanate (BGO) detectors covering the range from $\sim 200 \mathrm{keV}$ to $40 \mathrm{MeV}$. The detectors are arranged to collectively view the entire sky not blocked by the Earth.

In this paper, we use the BGO detector viewing the Sun to observe the nuclear line and continuum emission from solar flare SOL2010-06-12T00:57. Each BGO detector is a cylinder of length and diameter $12.7 \mathrm{~cm}$, viewed by a photomultiplier tube at each end. Its effective area for detecting photons ranges from $160 \mathrm{~cm}^{2}$ to $200 \mathrm{~cm}^{2}$, depending on energy and direction of incidence. Importantly for measuring solar flare nuclear lines, the high- $Z$ of bismuth, the high density of BGO, and the large volume of the GBM BGO detectors result in a high probability for absorbing the full incident photon energy: $\sim 67 \%$ at $1 \mathrm{MeV}$, $50 \%$ at $3 \mathrm{MeV}$, and $40 \%$ at $10 \mathrm{MeV}$.

Several types of data are produced by the GBM. There are two temporally binned types, Continuous Time (CTIME) and Continuous Spectroscopy (CSPEC), as well as Time-Tagged Events (TTE). For intense solar flares, TTE data are likely to be lost due to a bandwidth limit between the GBM and the spacecraft solid-state recorders. Therefore for solar-flare spectral analysis the appropriate data type is CSPEC. CSPEC has 128 quasi-logarithmic energy bins and $4.096 \mathrm{~s}$ temporal resolution, which is improved to $1.024 \mathrm{~s}$ when the flight software triggers on a statistically significant rate increase.

The detector performance was measured before launch using X-rays from the BESSY synchrotron radiation facility, radioactive isotopes including the ${ }^{12} \mathrm{C}$ line at $4.43 \mathrm{MeV}$ (from an ${ }^{241} \mathrm{Am} /{ }^{9} \mathrm{Be}$ source), and $\gamma$-rays up to $17.6 \mathrm{MeV}$ produced via $(\mathrm{p}, \gamma)$ reactions using a Van de Graaff accelerator. These data have been used to calibrate the gain, resolution, and response of the detectors (Bissaldi et al. 2009; Meegan et al. 2009). The automatic gain software measures and adjusts the gains of the BGO detectors using the $2.223 \mathrm{MeV}$ line present in the background from capture of cosmic-ray produced neutrons on $\mathrm{H}$ in the spacecraft's propellant. In order to improve the gain solution for analysis of the June 12 flare data we measured the centroid of the solar neutron-capture line. This resulted in a $1 \%$ gain change that is small relative to the $\sim 8 \%$ resolution of the GBM at the $2.223 \mathrm{MeV}$ line. The best-fit energy of the positron annihilation line $(0.511 \mathrm{MeV})$ from the flare was found to be 0.530 $\pm 0.007 \mathrm{MeV}$, in disagreement with fits of the instrumental background line. However, both lines are superimposed on strong continua making it difficult to determine the lines' peak position. Further investigation of the BGO calibration below $1 \mathrm{MeV}$ is planned, particularly since TGF spectra also show a shifted positron annihilation line (Briggs et al. 2011).

Spectral fitting is performed using the forward-folding technique with an assumed parameterized photon spectrum folded through a detector response matrix (DRM) to produce a counts spectrum; the parameters are adjusted to obtain the best fit to the observed counts spectrum. The DRM is based on Geant4 simulations of the GBM detectors and mass model of the satellite and has been validated by comparison with the calibration data (Hoover et al. 2008).

\section{LAT CAPABILITY FOR FLARE OBSERVATIONS}

The LAT is a pair-conversion telescope designed to detect gamma rays from $20 \mathrm{MeV}$ up to more than $300 \mathrm{GeV}$ (Atwood et al. 2009). It is made up of a $4 \times 4$ matrix of identical towers, each one comprised of a tracker with layers of Silicon Strip Detectors (SSD) alternating with foils of high-Z converter (tungsten), and a calorimeter with $\log$ s of CsI arranged in a "hodoscopic" configuration so that the energy deposition is imaged in three dimensions. The array of towers is surrounded by an Anti-Coincidence Detector (ACD) made up of 89 plastic scintillator tiles with a $5 \times 5$ array on the top and 16 tiles on each of the four sides. To reduce the impact of the selfveto events caused by calorimeter back-splash, each ACD tile is typically $<1000 \mathrm{~cm}^{2}$, depending on its position in the array. A $\gamma$-ray passes through the ACD with small probability of interaction and can convert into an electron-positron pair that is tracked in the SSD. The energy of a photon below about $100 \mathrm{MeV}$ can be estimated by multiple-Coulomb scattering of the electrons in the tungsten converters and SSD. Energies of higher-energy photons are measured by "total" absorption or modeling the shower profile in the calorimeter. In the standard Fermi sky-survey mode the spacecraft rocks $50^{\circ}$ north and south in celestial declination from the zenith so that each region of the sky is viewed for $\sim 30-40$ minutes every two orbits; therefore the LAT's large aperture $(2.4 \mathrm{sr})$ and effective area provides the capability to sensitively monitor solar activity with a duty cycle of $15 \%-20 \%$.

The ACD is used to reject the large background of charged cosmic-radiation and secondaries from the spacecraft and Earth's atmosphere. The threshold for this veto is nominally set at $45 \%$ of the amplitude of a minimizing ionizing singly charged particle traversing a tile in the ACD (i.e., set to $\sim 800 \mathrm{keV}$ ). If this threshold is exceeded and if the ACD tile hit was adjacent to a tracker tower that caused the event to trigger, the event will be vetoed unless a sufficiently high energy is deposited in the calorimeter ( $>100 \mathrm{MeV}$ in one or more crystals). This veto inhibition ensures that backsplash does not cause very high-energy $\gamma$-rays to self-veto. Of the vetoed events, $2 \%$ are telemetered to the ground for diagnostic purposes.

Solar flares can emit intense fluxes of tens of keV X-rays. About $20 \%$ of the X-rays at these energies can penetrate the thermal blanket and micrometeoroid shield and reach the ACD, depositing part of their energy in the illuminated tile.

Several of these photons can arrive within the $0.4 \mu \mathrm{s}$ anticoincidence veto shaping time (pulse pile-up) to yield a high total-energy loss. It is possible in the largest of flares that this energy loss could exceed the $800 \mathrm{keV}$ veto threshold and information about a valid photon event would not be transmitted 


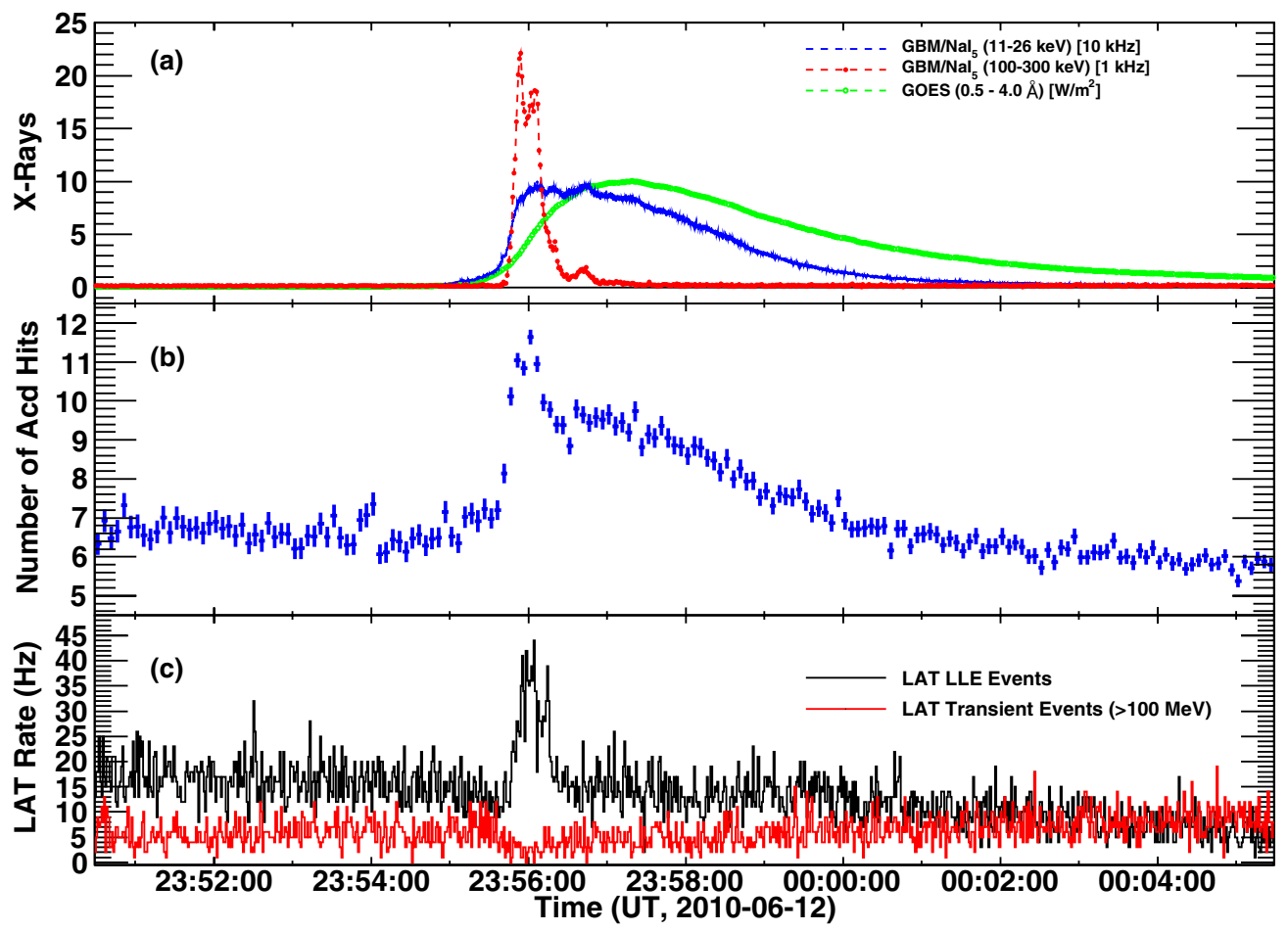

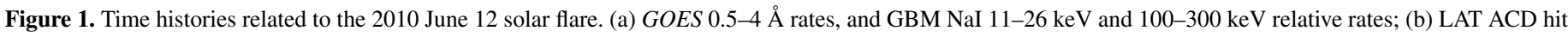

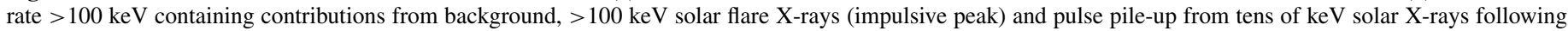
the NaI 11-26 keV profile in (a); and (c) LLE and LAT Transient Class event rates.

(A color version of this figure is available in the online journal.)

to the ground. None of the solar flares detected to date have been sufficiently intense to cause this data loss to occur.

Pulse pile-up of hard X-rays from some flares has, however, affected the ground analysis of LAT data, in the classification of events as $\gamma$-rays or background. ACD tile hits are registered (i.e., included in an event) whenever there is $>100 \mathrm{keV}$ energy deposition integrated over the $\sim 3 \mu$ s peaking time of the frontend amplifier (as opposed to the $800 \mathrm{keV}$ threshold for an ACD veto). Pulse pile-up during even modest flares generates such tile hits. In order to achieve the highest sensitivity for studying celestial sources, the current LAT ground-processing software rejects $\gamma$-ray events with a high ratio between the number of ACD tile hits (i.e., the number of tiles with energy deposition $>100 \mathrm{keV}$ ) and the energy measured by the calorimeter. Thus, $\gamma$-rays arriving during relatively intense flares have a high likelihood of being rejected from the standard LAT data products. This is the case for the 2010 June 12 flare as we will discuss in Section 4.2 below.

As a consequence of this feature, the LAT Low-Energy (LLE) technique (Pelassa et al. 2010) was adapted for analyzing data during this flare. LLE event selection uses less discriminating criteria than the standard ground-processing and is not affected by ACD tile hits $>100 \mathrm{keV}$. The primary requirement is that the candidate $\gamma$-ray event have at least one track and a reconstructed energy larger than $30 \mathrm{MeV}$. Due to these less discriminating criteria the off-axis angle for LLE events can be as large as $\sim 80^{\circ}$ compared with $\sim 68^{\circ}$ in the standard LAT data products. In addition, only $\gamma$-rays whose estimated arrival directions were within $20^{\circ}$ of the Sun are included for analysis. The $20^{\circ}$ angular restriction was chosen based on Monte Carlo simulations of the LAT point-spread function at these energies. A time series of events is then constructed from which background intervals on either side of the flare are defined and a linear or quadratic interpolation is used to estimate the background during the flare. A DRM for the solar location during the flare is created using a custom Monte Carlo simulation. By passing candidate photon models through the DRM, we then fit the background-subtracted data using a version of rmfit $3.4,{ }^{53}$ customized for the specific solar flare, and the $O S P E X^{54}$ analysis packages.

Using a Monte Carlo analysis, we estimate that the energy resolution for a source at $\sim 75^{\circ}$ off-axis in our LLE analyses is about $40 \%$. We also estimate $\sim 15 \%$ and $\sim 10 \%$ systematic uncertainties in the LLE fluxes $>30$ and $100 \mathrm{MeV}$, respectively, based on studies of the Vela pulsar. The uncertainty $>100 \mathrm{MeV}$ is consistent with that found using the standard Fermi analysis tools applied to the pulsar (Abdo et al. 2010).

\section{JUNE 12 FLARE OBSERVATIONS}

The GOES M2-class X-ray flare commenced with some lowlevel activity on 2010 June 12 at 00:30 UT. Although both RHESSI and Fermi observed the flare, here we only discuss the Fermi observations because RHESSI was not pointed at the Sun during the flare. The flare occurred with Fermi in sunlight and during a relatively low-background portion of its orbit. As seen in Figure 1(a) the 10-26 keV emission recorded by the GBM NaI detectors commenced around 00:55 UT and rose precipitously about $40 \mathrm{~s}$ later; for comparison we also plot the GOES 0.5-4 A profile and note that this emission is dominated by $3 \mathrm{keV}$ thermal photons as is reflected in its slower rise and extended tail. The GBM burst algorithm triggered on the flare at 00:55:05.64 UT and put the instrument in a high-data rate

\footnotetext{
53 R. S. Mallozzi, R. D. Preece, \& M. S. Briggs, "RMFIT, A Lightcurve and Spectral Analysis Tool," Robert D. Preece, University of Alabama in Huntsville (2008): http://fermi.gsfc.nasa.gov/ssc/data/analysis/user/ 54 SolarSoft: http://www.lmsal.com/solarsoft/
} 
mode for the next 10 minutes. The $100-300 \mathrm{keV}$ time profile observed by the GBM's solar facing $\mathrm{NaI}$ detector is also plotted in Figure 1(a). It is clear that the emission peaks more sharply and ends sooner at higher X-ray energies. Most of the emission observed above $100 \mathrm{keV}$ occurred within an $\sim 50 \mathrm{~s}$ interval. The event as viewed in the GOES 1-8 $\AA$ channel ended about 01:30 UT. The flare originated from active region (AR) 11081 at approximately $\mathrm{N} 23^{\circ} \mathrm{W} 43^{\circ}$. White light emission observed by the Helioseismic and Magnetic Imager (HMI) on the Solar Dynamics Observatory (SDO) (Martínez Oliveros et al. 2011) in a single $45 \mathrm{~s}$ exposure, consistent in time with the hard X-ray emission, reveals two compact footpoints about $10^{4} \mathrm{~km}$ apart.

There was no evidence for significant dead time and/or pulse pile-up effects in the GBM BGO detector facing in the solar direction. Photons with energies up to $\sim 8 \mathrm{MeV}$ were detected by the GBM during the $50 \mathrm{~s}$ peak. At the time of the flare the spacecraft was rocking $50^{\circ}$ to the south so that the Sun was $76^{\circ}$ off-axis, close to the edge of the field of view (FOV) for LLE studies, and Earth's horizon was entering the FOV. The accompanying hard X-ray emission from the flare was detected in the LAT's ACD. In Figure 1(b), we plot the average number of ACD tile hits as a function of time. As discussed in the previous section, pulse pile-up from tens of keV hard X-rays exceeded the $100 \mathrm{keV}$ ACD hit threshold. This is reflected in the broad peak with a maximum near 00:57 UT that has a shape similar to the 11-26 keV emission observed by the GBM NaI detector. The impulsive peak in the ACD rate is also similar to that observed between 100 and $300 \mathrm{keV}$ by the GBM NaI detector. There is no evidence for an increase in the number of ACD vetoed events in orbit during the flare, indicating that pulse pile-up from hard $\mathrm{X}$-rays did not exceed the $800 \mathrm{keV}$ veto threshold. Thus, the overall valid event rate transmitted to the ground for processing was not affected by the ACD response. However, as shown by the red curve in Figure 1(c) there is no evidence for the flare in the well-screened standard LAT data products (shown in the figure are the events belonging to the "transient" event class; Atwood et al. 2009). If anything, we see a deficit of events in the standard analysis light curve which is a consequence of the high ACD hit rate $>100 \mathrm{keV}$, shown in Figure 1(b), that caused a significant increase in the number of events that failed the standard quality cut.

It is important to convincingly establish that any high-energy emission observed by the LAT originated at the flare site and was not due to artifacts from the high rates encountered by the instrument. The most compelling evidence for the solar flare origin is the map of events relative to the position of the Sun. Plotted in Figure 2(a) is the distribution of LLE-selected events with energies $>30 \mathrm{MeV}$ accumulated $30 \mathrm{~s}$ before and after the flare. The distribution is affected by the instrument FOV and by the removal of events near Earth's horizon. We reduce the contamination from the bright Earth limb selecting only the events with a reconstructed zenith angle less than $100^{\circ}$, but, due to the large point spread function at low energy, some residual contamination near the horizon may be due to this limb brightening.

In Figure 2(b), we plot the distribution accumulated from 00:55:40 to 00:56:30 UT during the flare. We note that the excess near the Sun's position is biased due to its location near the edge of the LAT's FOV. This is known as the "fisheye" effect, i.e., the tendency of events to be reconstructed with directions closer to the $z$-axis than they should (Thompson et al. 1993). This bias is relevant only for off-axis events, and it is particularly evident at low energies. The position of the centroid of the counts is

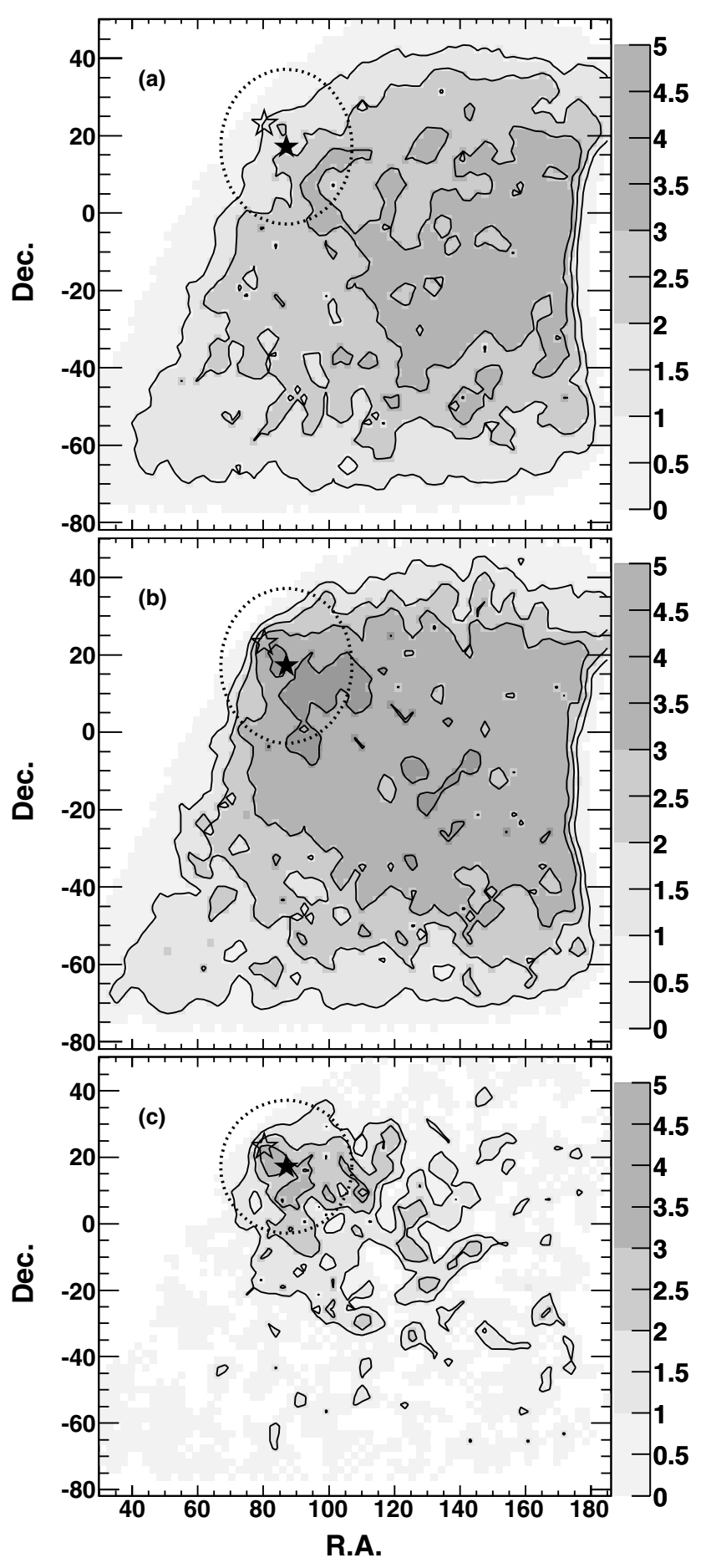

Figure 2. Angular distribution of $>30 \mathrm{MeV} \gamma$-rays relative to the position of the Sun detected using the LLE analysis. The open star shows the location of the Sun which is close to the is close to the edge of the LAT field of view. The filled star shows the shifted position due to the fisheye effect discussed in the text. The dotted curve depicts the $20^{\circ}$ region containing the events used in the analysis. (a) Average of background distribution taken $30 \mathrm{~s}$ before and after the flare. (b) Angular distribution of events observed between 00:55:40 and 00:56:30 UT. (c) Difference between angular distribution observed during the flare and the average background distribution. The distributions have been smoothed (with a Gaussian kernel) to reduce statistical fluctuations.

shown by the filled star (at R.A. $=84^{\circ} .39$, Decl. $=18^{\circ} .79$, $\mathrm{J} 2000$ ). It is important to note that in the routine LAT analyses the contribution to the overall exposure and photon counts from far off-axis events is negligible. In addition to this, owing to the scanning mode of observations with the LAT, persistent sources 


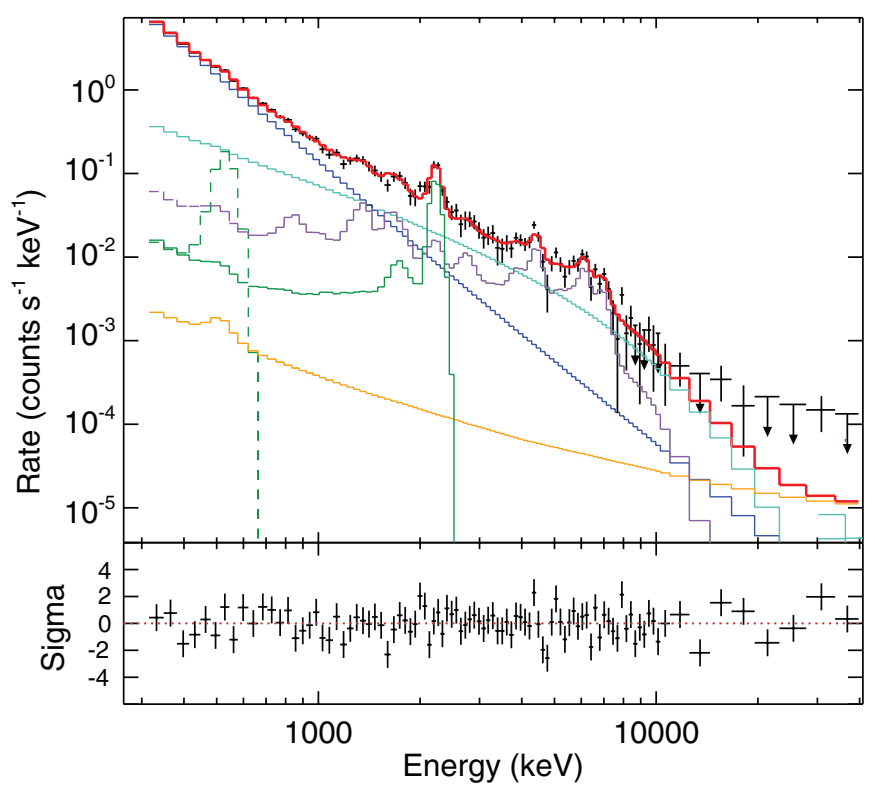

Figure 3. Background-subtracted BGO count spectrum accumulated between 00:55:40 and 00:56:30 UT. The spectrum has been fit by a simple power law (blue histogram) and a flatter power law with exponential cutoff (light green histogram) to model bremsstrahlung by electrons (see the text), and with a nuclear de-excitation component (purple) plus 0.511 and $2.223 \mathrm{MeV}$ lines (dashed and dark green), and a pion-decay (orange) component.

(A color version of this figure is available in the online journal.)

will have the great majority of their exposure at much smaller inclination angles.

In Figure 2(c), we plot the background-subtracted distribution observed during the flare. There appears to be significant $\gamma$-ray emission consistent with the reconstructed position of the Sun up to energies of $\sim 400 \mathrm{MeV}$. We have confirmed the observed $6^{\circ}$ shift in the position of the centroid from the solar position using a Monte Carlo study. Using the shift and identifying a $<20^{\circ}$ acceptance angle (dashed curve in the figure) for LLE events $>30 \mathrm{MeV}$ maximizes the signal/background. Events that meet these criteria make up the LLE rate (black curve) plotted in Figure 1(c). The overall rate decreased, especially after 01:00 UT because the defined aperture approached the exclusion region near Earth's limb and many events were consequently rejected. The $>30 \mathrm{MeV}$ LLE time profile during the flare is similar to the 100-300 keV NaI time history.

\subsection{GBM Spectroscopic Studies}

In order to obtain background-subtracted spectra, we used GBM/BGO spectra accumulated just before the flare and after 4 minutes following the flare for background in order to avoid times when there was significant $15-50 \mathrm{keV}$ hard X-ray emission. The background-subtracted GBM counts spectrum accumulated over a $50 \mathrm{~s}$ period (00:55:40-00:56:30 UT) during the impulsive phase of the flare is shown in Figure 3. Although the flare was relatively weak and the BGO detector has only moderate energy resolution, line features are clearly evident in the spectrum.

The spectrum has been fit with a photon model consisting of several components. We find that the electron-bremsstrahlung component has the shape of power law at low energies that hardens above several hundred $\mathrm{keV}$ and then rolls over in the $\mathrm{MeV}$ range. In this paper, we fit the bremsstrahlung spectrum with the sum of a low-energy power law and a flat power law with exponential cutoff in the $\mathrm{MeV}$ range (blue and light green
Table 1

Best-fitting Spectral Parameters

\begin{tabular}{|c|c|}
\hline Parameter & Value \\
\hline PL1 fluence at $300 \mathrm{keV}$ & $2.85 \pm 0.1 \gamma \mathrm{cm}^{-2} \mathrm{keV}^{-1}$ \\
\hline PL1 index & $3.31 \pm 0.09$ \\
\hline PL2 fluence at $300 \mathrm{keV}$ & $0.08 \pm 0.02 \gamma \mathrm{cm}^{-2} \mathrm{keV}^{-1}$ \\
\hline PL2 index & $\lesssim 1.2$ \\
\hline PL2 Exponential Energy & $2400 \pm 800 \mathrm{keV}$ \\
\hline $0.511 \mathrm{MeV}$ line fluence ${ }^{\mathrm{a}}$ & $11.3 \pm 2.5 \gamma \mathrm{cm}^{-2}$ \\
\hline 2.223 MeV line fluence ${ }^{\mathrm{a}}$ & $21.3 \pm 2.0 \gamma \mathrm{cm}^{-2}$ \\
\hline Nuclear line fluence & $23.5 \pm 2.5 \gamma \mathrm{cm}^{-2}$ \\
\hline Pion-decay fluence $(\mathrm{GBM})>200 \mathrm{keV}$ & $1.5 \pm 2.5 \gamma \mathrm{cm}^{-2}$ \\
\hline Pion-decay fluence $\left(\mathrm{LAT}^{\mathrm{b}}\right)>200 \mathrm{keV}$ & $0.62 \pm 0.07 \gamma \mathrm{cm}^{-2}$ \\
\hline Pion-decay fluence (LAT) $>100 \mathrm{MeV}$ & $0.13 \pm 0.015 \gamma \mathrm{cm}^{-2}$ \\
\hline PL3 fluence at $30 \mathrm{MeV}$ & $(9.2 \pm 2.0) \times 10^{-6} \gamma \mathrm{cm}^{-2} \mathrm{keV}^{-1}$ \\
\hline PL3 index & $1.9 \pm 0.2$ \\
\hline
\end{tabular}

Notes.

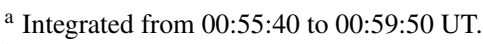

${ }^{\mathrm{b}}$ Computed by extrapolating to low energies the model that best fits LAT data.

curves, respectively, in Figure 3). Such a complex shape has been observed before in the spectra of several flares detected by $S M M$ and RHESSI (G. H. Share \& R. J. Murphy, in preparation). McTiernan \& Petrosian (1990) have found that the magnitude of the hardening above a few hundred $\mathrm{keV}$ observed in some flares is larger than that expected for an electron spectrum following a single power law. Rieger \& Marschhäuser (1991) describe flattening in the $\mathrm{MeV}$ range followed by rollovers above several tens of $\mathrm{MeV}$ in the spectra of some flares. Such features cannot be explained by transport effects alone (Petrosian et al. 1994) and must be produced by the acceleration mechanism. Park et al. (1997) show that these features can be explained by models based on stochastic acceleration by turbulence once loss mechanisms are properly included. Whether such models can explain the 2010 June 12 bremsstrahlung spectrum requires more study.

The nuclear de-excitation lines and continua are represented by a template based on a detailed study of nuclear gammaray production from accelerated-particle interactions with elements found in the solar atmosphere (Murphy et al. 2009). Such templates depend on the assumed ambient composition and accelerated-particle composition and spectrum. The BGO spectral data for this flare are inadequate to distinguish among templates derived for different ambient abundances, particle spectra and angular distributions. For this reason, we used a arbitrary templates based on earlier studies (G. H. Share \& R. J. Murphy, in preparation). The accelerated particles were assumed to interact in a thick target with a coronal composition (Reames 1995), but with ${ }^{4} \mathrm{He} / \mathrm{H}=0.1$. We do not take into account transport effects but instead assume that the accelerated particles have a power-law differential spectrum $\left(d N / d E \propto E^{\beta}\right.$ with $\beta=-4$ ), coronal elemental abundance (but with an accelerated $\alpha / \mathrm{p}$ ratio of 0.2 ), and an angular distribution that is isotropic in the downward hemisphere. As we mentioned above our results are not sensitive to the assumed compositions. The photon model also includes Gaussians representing the 0.511 and $2.223 \mathrm{MeV}$ positron-electron annihilation and neutron-capture lines, respectively, and a pion-decay spectral component.

We present the best-fitting spectral parameters in Table 1, along with estimates of their $1 \sigma$ uncertainties. We list fluences obtained by integrating over the $50 \mathrm{~s}$ time period, except for the 
0.511 and $2.223 \mathrm{MeV}$ lines. The $0.511 \mathrm{MeV}$ line originates from radioactive decays (Kozlovsky et al. 1987, 2004) with half lives that can extend up to hours, in addition to a prompt component from positron annihilation following decay of positively charged pions. The neutron-capture line is also delayed because of the time required for the neutrons to slow down in the solar atmosphere and photosphere and be captured on $\mathrm{H}$, forming deuterium with the release of $2.223 \mathrm{MeV}$ of binding energy from the mass excess. For these two lines, we have fit the backgroundsubtracted spectrum integrated over a total of $250 \mathrm{~s}$.

The low-energy power law (PL1) is well defined by the fit. The higher-energy power law (PL2) with exponential rollover is not well defined because it competes with the nuclear deexcitation line spectrum. However, our studies indicate that this component is required to provide an acceptable fit to the June 12 flare spectrum. With this component present, the fit is acceptable at the $10 \%$ confidence level based on the $\chi^{2}$ statistic; without it, the fit is rejected $(0.001 \%$ confidence level $)$. There is no evidence for pion-decay emission in the BGO spectrum. As can be seen in Table 1, assuming an hadronic origin, the LATmeasured pion-decay fluence that we discuss in the next section is about a factor of 10 lower than detectable by the GBM BGO detector.

\subsection{Combined LAT and GBM Spectroscopic Studies}

We have obtained a background-corrected LAT count spectrum $>30 \mathrm{MeV}$ accumulated during the $50 \mathrm{~s}$ period 00:55:4000:56:30 UT using the LLE data plotted in Figure 1(c). This spectrum revealed flare emission up to an energy of $\sim 400 \mathrm{MeV}$. The fundamental question is: what is the origin of this emission? The nuclear line emission observed with the GBM implies the presence of accelerated ions up to at least $50 \mathrm{MeV}$ nucleon $^{-1}$. It is possible that the flare-accelerated proton spectrum extended up to the $\sim 300 \mathrm{MeV}$ threshold for pion production. Alternatively, it is possible that the LAT emission is from electron bremsstrahlung, either from an extension to high energies of the electron spectrum producing the X-ray bremsstrahlung observed in the GBM or from an additional hard electron component. One possible way to resolve this ambiguity is to jointly fit the GBM and LAT spectra assuming different origins for the LAT emission.

In Figure 4, we plot the background-subtracted photon spectrum from 0.3 to $400 \mathrm{MeV}$ including both the GBM and LAT data. We made two fits to the joint data sets, one assuming that the observed LAT emission was from pion-decay radiation (the top panel) and the other assuming that it was from a hard powerlaw spectrum of electron bremsstrahlung (the bottom panel). Based on the statistical quality of the fits to the LAT spectrum we cannot distinguish between the two emission models. In addition we cannot constrain the origin of the emission for this event by extrapolating the models into the GBM energy range; however we note that for a stronger flare we might be able to rule out a power-law model. The choice of model also has only a small effect on the parameters listed in Table 1 derived from fits to the $0.3-40 \mathrm{MeV}$ GBM data. Also plotted in the figure are the extensions into the LAT energy range of the power-law and cutoff power-law components derived from the fits to the GBM data. The intensities of these components fall at least an order of magnitude below the LAT measurements and therefore do not make a significant contribution to the solar emission observed by the LAT.

Even though we cannot statistically distinguish between a pion-decay or electron-bremsstrahlung origin for the ob-

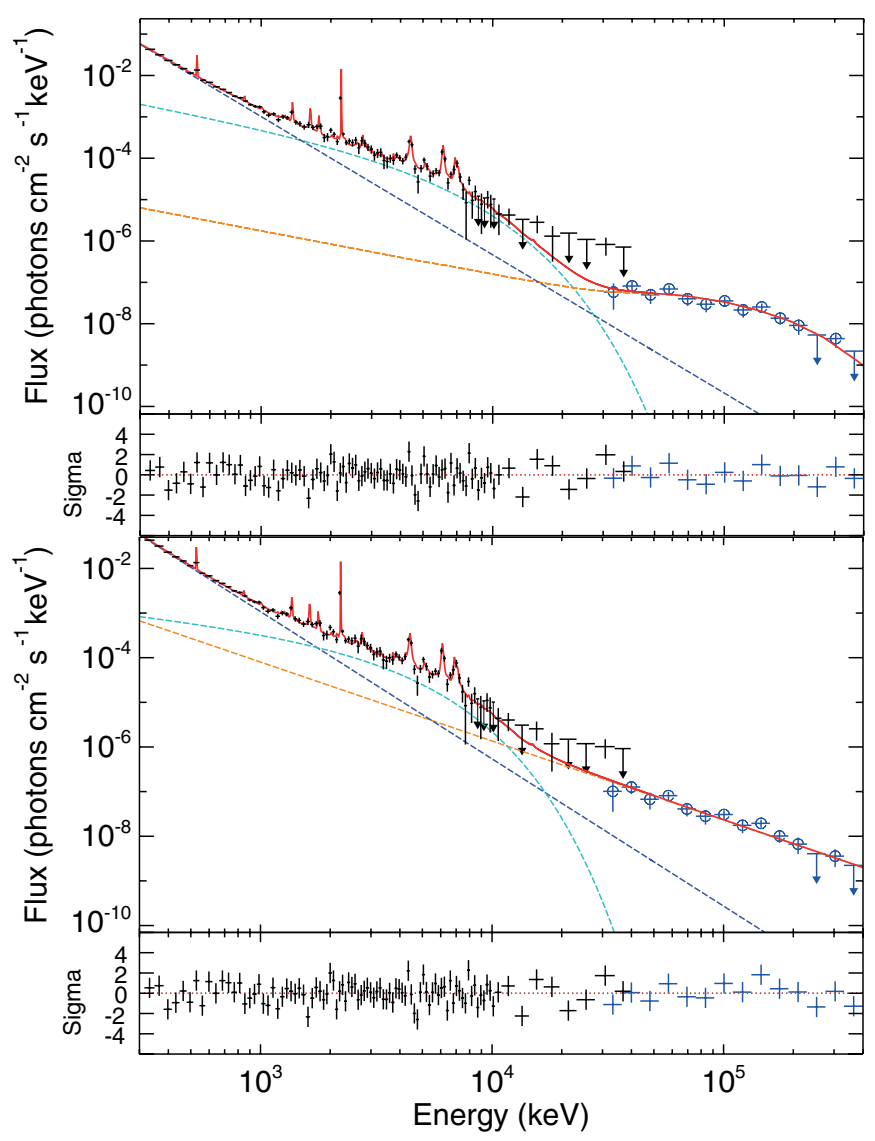

Figure 4. Combined GBM/LAT photon spectrum accumulated between 00:55:40 and 00:56:30 showing the best total fit using the same components as in Figure 3 plus an additional component for the LAT emission. The upper panel shows a pion-decay fit to the LAT spectrum; alternatively the lower panel shows a power-law fit, presumedly representing a third electron bremsstrahlung component. Note that because this is a photon representation the lines are plotted at their intrinsic resolution and appear to be more significant than they really are.

(A color version of this figure is available in the online journal.)

served LAT emission, we can obtain the best-fitting parameters for these components. If the LAT emission is from electron bremsstrahlung, we have shown that it cannot be a simple extension of the low-energy bremsstrahlung components that we determined from fits to the GBM data; it must be from a distinct population of electrons extending to energies of several hundred $\mathrm{MeV}$. We have determined the power-law parameters (PL3) of the fitted bremsstrahlung spectrum and list them in Table 1. However, this third high-energy electron component cannot be produced by the acceleration models mentioned Section 4.1 above, which produce spectra that steepen beyond tens of $\mathrm{MeV}$ due to synchrotron energy losses that increase with energy (see Park et al. 1997), and must have a quite different origin. Consequently, we believe that this is a less likely scenario than the hadronic model.

Assuming that the LAT emission is from hadronic interactions, we have fit the LAT spectrum with calculated piondecay spectra produced by accelerated ions having differential power-law indices from -2.5 to -7.5 . With $67 \%$ confidence (based on $\chi^{2}$ ) we conclude that the spectrum of accelerated ions responsible for the pion-decay emission must be steeper than a power law with index -4.5 . We note that there is no change in the quality of the fits for indices steeper than -5 due to limited statistics $>400 \mathrm{MeV}$. We list the fluence of pion-decay photons 


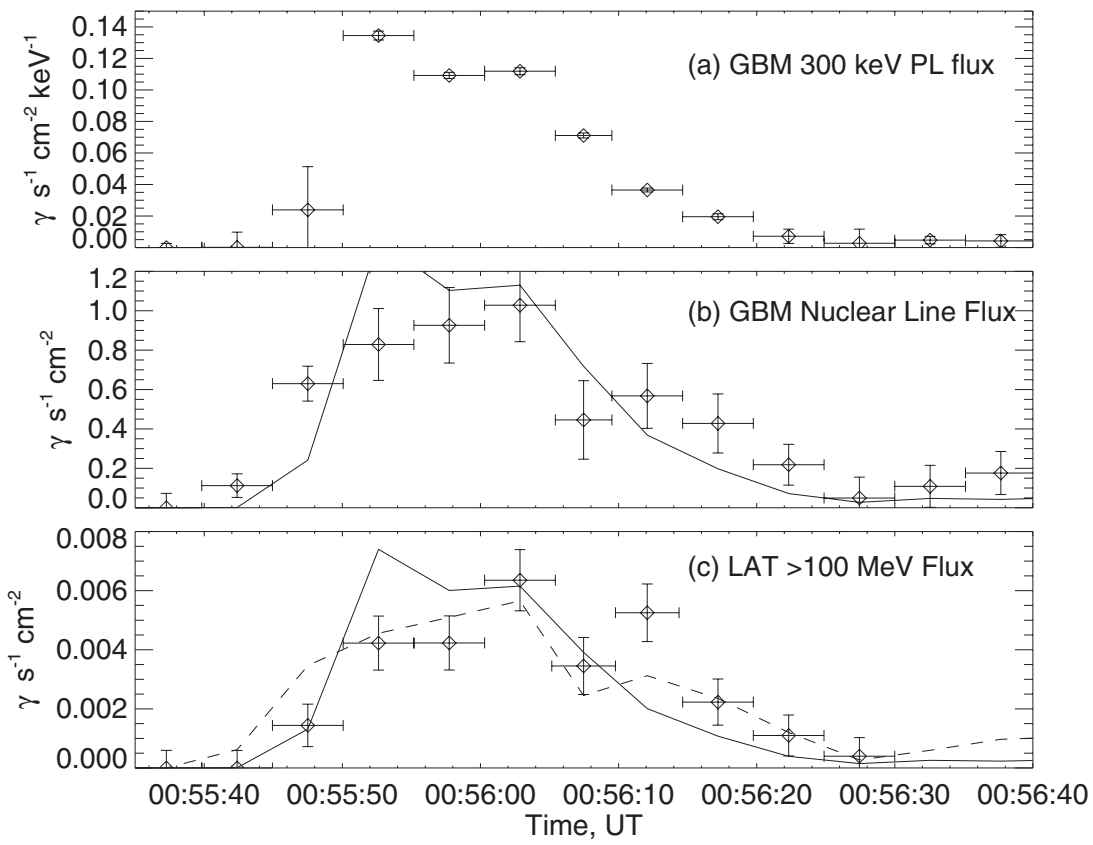

Figure 5. Time profiles of the (a) bremsstrahlung differential flux at $300 \mathrm{keV}$ (GBM), (b) total nuclear de-excitation line flux (GBM), and (c) integral pion-decay flux $>100 \mathrm{MeV}$ (LAT). Solid curves overplotted in (b) and (c) are the arbitrarily normalized bremsstrahlung flux plotted in (a); dashed curve overplotted in (c) is arbitrarily normalized nuclear line flux from panel (b).

$>200 \mathrm{keV}$ derived from these fits in Table 1 . This fluence is a factor of 10 below the limit derived from fitting the GBM BGO data up to $40 \mathrm{MeV}$, illustrating the significantly greater sensitivity of the LAT instrument.

We can use the results of our GBM and LAT spectral analyses to obtain information on ions accelerated in the impulsive phase of the June 12 flare. Murphy et al. (1997) have described how parameters derived from integrated spectroscopic fits and temporal studies can be used to obtain this information. We first use the nuclear de-excitation line, neutron-capture line, and pion-decay fluences listed in Table 1 to estimate the overall shape of the accelerated ion spectrum. These three emissions are produced by accelerated ions within distinct energy ranges: $\sim 5-20 \mathrm{MeV}$ for the de-excitation lines, $\sim 10-50 \mathrm{MeV}$ for the neutron capture line, and $>300 \mathrm{MeV}$ for the pion-decay emission. Ratios of these emissions therefore determine the relative numbers of accelerated ions in the associated energy ranges. We then obtain spectral indices across these energy ranges by comparing measured ratios with ratios from theoretical calculations (Murphy et al. 1987, 2005, 2007) based on updated nuclear cross sections.

If we assume that the LAT emission $>30 \mathrm{MeV}$ was entirely due to pion-decay emission, then we estimate that the flareaccelerated ion spectrum was consistent with a series of power laws, softening with energy, with indices of $\sim-3.2$ between $\sim 5-50 \mathrm{MeV}, \sim-4.3$ between $\sim 50-300 \mathrm{MeV}$, and softer than $\sim-4.5$ above $300 \mathrm{MeV}$. However, these calculations assume that these individual power laws continued without break to high energies, which cannot be the case where the spectrum softens with increasing energy. We will describe a more refined representation of the accelerated proton spectrum in a forthcoming publication.

\subsection{Combined GBM and LAT Timing Studies}

The combined GBM and LAT observations also provide us with the opportunity to study acceleration and transport in this impulsive flare. In Figure 5, we plot 5 s resolution time profiles of the fitted bremsstrahlung and nuclear de-excitation line fluxes from the GBM and the $>100 \mathrm{MeV}$ flux observed by the LAT. For purposes of comparison, we have plotted the bremsstrahlung profile over the nuclear and LAT $>100 \mathrm{MeV}$ histories in panels (b) and (c), respectively. We have also plotted the nuclear profile over the LAT $>100 \mathrm{MeV}$ history. The early peaking of the bremsstrahlung suggests that the higher-energy emissions were delayed by a few seconds and a cross-correlation study indicates that the overall lag between the LAT $>100 \mathrm{MeV}$ flux and the GBM $300 \mathrm{keV}$ bremsstrahlung flux is $\sim 3 \mathrm{~s}$.

This delay warranted a higher-time resolution comparison of $100-500 \mathrm{keV}$ GBM and LAT $>30 \mathrm{MeV}$ counting rates rather than fitted fluxes. The $100-500 \mathrm{keV}$ band is dominated by electron bremsstrahlung as can be seen in Figure 3. In Figure 6, we plot GBM/BGO $100-500 \mathrm{keV}$ rates at $320 \mathrm{~ms}$ resolution with 3 s LAT/LLE $>30 \mathrm{MeV}$ rates overplotted. The hard $\mathrm{X}$-ray profile reveals the presence of a clearly separated initial peak along with other structures. The onset of the $>30 \mathrm{MeV}$ emission appears to be $\sim 3$ s following the bremsstrahlung and rises to a peak about $10 \mathrm{~s}$ after the $100-500 \mathrm{keV}$ peak. The LLE profile appears to reflect the double-peaked bremsstrahlung profile with a delay of about $10 \mathrm{~s}$. From a cross-correlation analysis of the two profiles plotted in Figure 6, we find that the $>30 \mathrm{MeV}$ emission lags the bremsstrahlung by $6 \pm 3 \mathrm{~s}$. There are two fundamental implications of the time profiles in Figure 6: (1) protons and/or electrons began reaching energies above $100 \mathrm{MeV}$ within a few seconds of the time it took to accelerate electrons to energies of hundreds of $\mathrm{keV}$; and (2) the overall acceleration time scale of the $>100 \mathrm{MeV}$ particles is similar to that observed in hundreds of $\mathrm{keV}$ electrons, but delayed by about $10 \mathrm{~s}$.

\subsection{Search for $>100 \mathrm{MeV}$ Emission Following the Flare}

The Fermi LAT detects quiescent emission from the Sun on a near daily basis (Abdo et al. 2011). This emission comes 


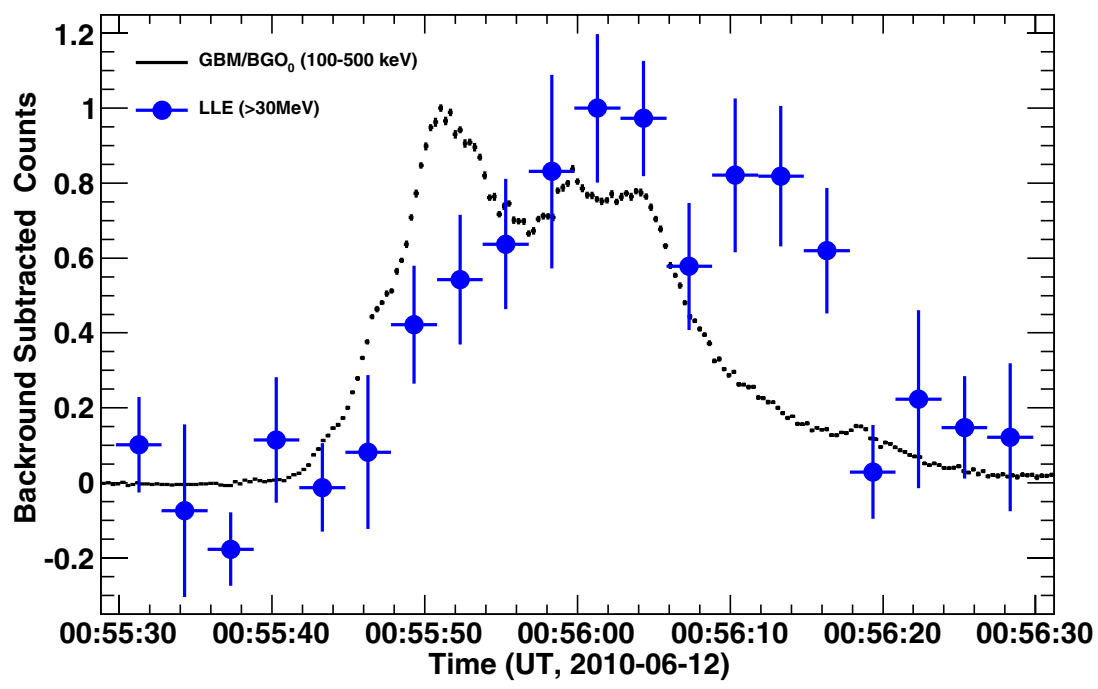

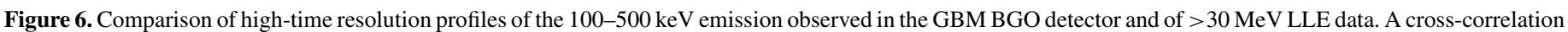
analysis indicates that the high-energy $\gamma$-ray emission had an overall lag of $6 \pm 3$ s relative to the bremsstrahlung.

(A color version of this figure is available in the online journal.)

from cosmic-ray proton interactions in the solar atmosphere and photosphere, and from Compton scattering of cosmic-ray electrons on solar blackbody photons. The LAT is therefore a sensitive monitor of temporally extended solar-flare emission such as detected by CGRO EGRET experiment following the 1991 June 11 flare (Kanbach et al. 1993; Rank et al. 2001). We therefore studied the emission within $15^{\circ}$ of the Sun in the hours preceding and following the flare. The standard LAT data products were used in the analysis, which modeled the region around the Sun including all sources in the Second Fermi LAT Source Catalog (The Fermi-LAT Collaboration 2011), isotropic and Galactic diffuse emissions, and spatially extended Compton-scattered solar photons discussed above.

In Figure 7, we plot 95\% confidence limits on the $>100 \mathrm{MeV}$ flux from the solar disk in $\sim 30$ minutes exposures every two orbits from $6 \mathrm{hr}$ before the flare to $22 \mathrm{hr}$ after it. We note that the Sun was outside the FOV for LAT standard-product analysis during the flare, but its $\gamma$-ray emission could be studied in the orbits just before and after the flare. During this time period the Moon passed within $10^{\circ}$ of the Sun and its flux of $\sim 1 \times 10^{-6} \gamma \mathrm{cm}^{-2} \mathrm{~s}^{-1}$ made a significant contribution to the measured solar fluxes because we did not include it in the model of the source region. There is no evidence for an increase in the solar emission following the time of the flare, which is denoted by the peak flare flux $>100 \mathrm{MeV}$. This peak flux is about 1000 times higher than the plotted upper limits. We wish to compare this to the time extended $>100 \mathrm{MeV}$ emission observed by EGRET following the 1991 June 11 flare (Kanbach et al. 1993; Rank et al. 2001). But EGRET was saturated at the peak of the flare and therefore its time history cannot be normalized to the $>100 \mathrm{MeV}$ LAT peak flux of the June 12 flare. However, after the peak of the flare the ratio of the $>100 \mathrm{MeV}$ EGRET flux to the $2.223 \mathrm{MeV}$ neutron-capture line measured by COMPTEL, which operated normally throughout the June 11 flare, was relatively constant. We therefore used the COMPTEL time history as a proxy for the EGRET $>100 \mathrm{MeV}$ photon fluxes. The time profile of the COMPTEL $2.223 \mathrm{MeV}$ neutron-capture line flux (Share et al. 1997), normalized to the peak $>100 \mathrm{MeV}$ LAT June 12 flux, is shown in the Figure 7. We see that during the first 30 minute exposure following the flare, the LAT upper limit is a factor of $\sim 20$ below the value expected if there had been comparable extended emission similar to that found on 1991 June 11.

\section{SUMMARY AND DISCUSSION}

The 2010 June 12 flare was the first in Cycle 24 to be observed to emit nuclear $\gamma$-rays. It was also the first flare detected by the Fermi LAT at energies above $30 \mathrm{MeV}$. The hard X-ray and nuclear line radiation was observed both by the Fermi GBM and RHESSI spectrometers. In this paper, we only analyzed GBM data because RHESSI was offset from the Sun to study the Crab Nebula during the time period of the flare; this affected knowledge of the instrument response.

The fact that the flare emitted detectable $\gamma$-rays at all is surprising because its peak soft X-ray emission only reached a GOES M2 level. However, Shih et al. (2009) and others have shown that $\gamma$-ray line fluences are only weakly correlated with GOES soft X-ray emission but are strongly correlated with electron bremsstrahlung fluences $>300 \mathrm{keV}$. This is true for the June 12 flare as we find that the measured bremsstrahlung and $2.223 \mathrm{MeV}$ fluences are consistent with the established correlation.

The flare originated from a compact region and its hard X-ray emission only lasted $50 \mathrm{~s}$. Figure 6 reveals striking information about the processes that accelerate protons and/or electrons to energies of hundreds of $\mathrm{MeV}$. We find that although some of the particles reach energies $\gtrsim 100 \mathrm{MeV}$ within about $3 \mathrm{~s}$, the bulk of these particles reach such high energies following a delay of about $10 \mathrm{~s}$. This is revealed in the delayed double-peaked time structure $>30 \mathrm{MeV}$ that is similar to what is observed in hard X-rays.

In Table 1, we list the best-fitting parameters from our fits to the GBM and LAT spectra. These include the amplitudes (at $300 \mathrm{keV}$ ) and indices of two power-law continua observed by the GBM between $300 \mathrm{keV}$ and $8 \mathrm{MeV}$. The first is an extension of the hard X-ray spectrum observed by the GBM NaI detectors. The second appears to be a hard power law with an exponential cutoff energy near $2.5 \mathrm{MeV}$. Although the GBM only has moderate spectral resolution, it was able to measure the fluences of the $0.511 \mathrm{MeV}$ annihilation and $2.223 \mathrm{MeV}$ neutron capture lines, and the total nuclear de-excitation emission. There was no 


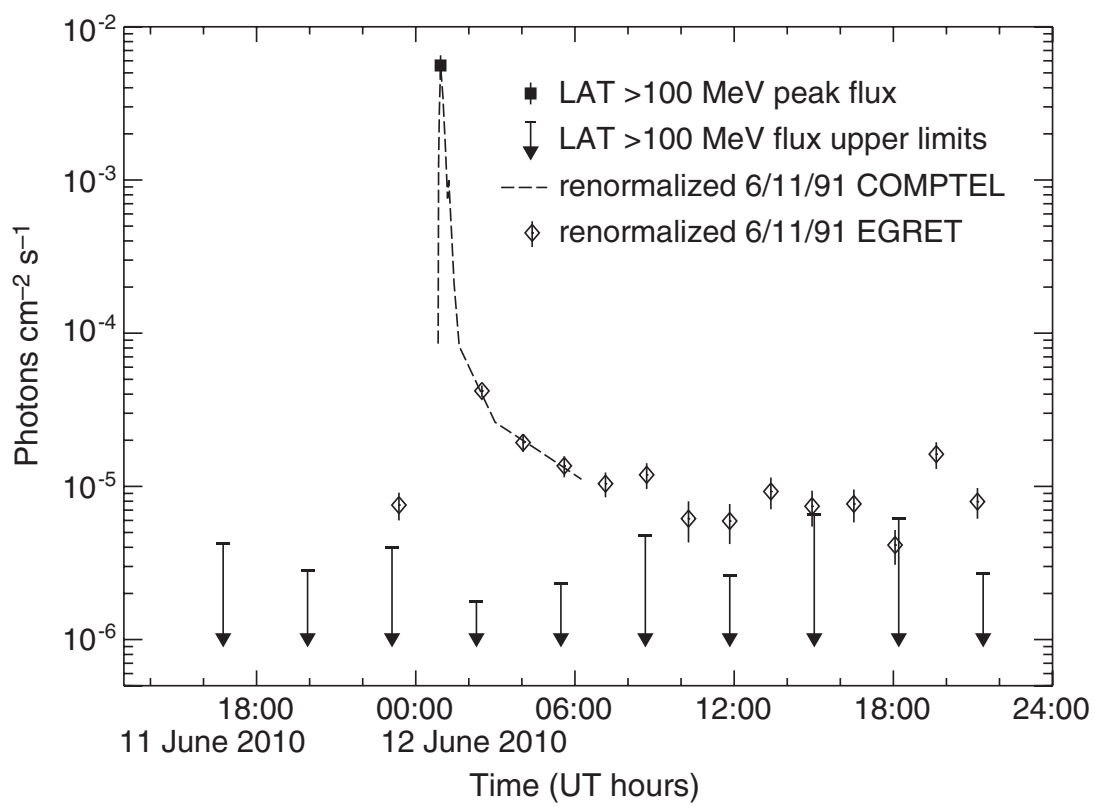

Figure 7. $95 \%$ confidence upper limits of $>100 \mathrm{MeV}$ solar $\gamma$-rays measured by LAT within $7 \mathrm{hr}$ preceding and $22 \mathrm{hr}$ following the $2010 \mathrm{June} 12 \mathrm{flare}$. Dashed line is the 1991 June 11 time history of the $2.223 \mathrm{MeV}$ neutron-capture line observed by COMPTEL experiment and diamonds are EGRET $>50 \mathrm{MeV}$ data both normalized to the peak $>100 \mathrm{MeV}$ flux measured by the LAT for the June 12 flare. The 2010 June 12 flare does not show extended emission like the 1991 June 11 flare observed with EGRET.

evidence in the GBM data for flare emission above about $8 \mathrm{MeV}$. In contrast the LAT detected significant continuum emission from $\sim 30$ to $400 \mathrm{MeV}$ about an order of magnitude below the GBM upper limits. This reflects the excellent sensitivity of the LAT for observing solar flares. This radiation could be either pion-decay emission or primary electron bremsstrahlung. In Table 1, we list the measured pion-decay fluences $>200 \mathrm{keV}$ and $>100 \mathrm{MeV}$. We also list the parameters of a high-energy power-law bremsstrahlung component that fits the LAT data equally well. Theoretical arguments made in Section 4.2 weigh against an electron origin for the emission observed by the LAT. Under the assumption of an hadronic origin for the LAT emission and using $\gamma$-ray line measurements by the GBM, we have estimated the shape of the accelerated-ion spectrum that could have produced the combined spectrum.

We have also set significant constraints on any time-extended $>100 \mathrm{MeV}$ emission. Our limit on the $>100 \mathrm{MeV}$ flux of photons is about an order of magnitude below what would have been expected if the decay followed that observed in the wellstudied 1991 June 11 flare (Kanbach et al. 1993; Rank et al. 2001). Thus, there is no evidence for precipitation of trapped flare particles, particles accelerated in magnetic loops after the impulsive phase, particles accelerated in coronal mass ejection associated with reconnection sheets (Ryan 2000), or particles sharing the same origin as the Solar Energetic Particles (SEPs) observed in space (Ramaty et al. 1987; Cliver et al. 1993). We estimate from white light measurements of the 1991 June 11 flare (Sakurai et al. 1992) that the footpoint separation was $\sim 2.5 \times 10^{4} \mathrm{~km}$, about 2.5 times larger than the June 12 flare. It is possible that longer coronal loops are necessary for timeextended acceleration and/or trapping of protons.

This paper primarily addresses only the $\gamma$-ray observations of the 2010 June 12 flare. Other studies are currently in progress involving hard X-ray observations of the flare by the Fermi GBM NaI detectors and comparisons of the characteristics of the ion and electron populations at the Sun and in space and at Earth.
We thank the referee for suggesting a more detailed examination of the delay between the hard X-ray bremsstrahlung and $>30 \mathrm{MeV}$ emission observed by LAT. The Fermi LAT Collaboration acknowledges generous ongoing support from a number of agencies and institutes that have supported both the development and the operation of the LAT as well as scientific data analysis. These include the National Aeronautics and Space Administration and the Department of Energy in the United States, the Commissariat à l'Energie Atomique and the Centre National de la Recherche Scientifique/Institut National de Physique Nucléaire et de Physique des Particules in France, the Agenzia Spaziale Italiana and the Istituto Nazionale di Fisica Nucleare in Italy, the Ministry of Education, Culture, Sports, Science and Technology (MEXT), High Energy Accelerator Research Organization (KEK) and Japan Aerospace Exploration Agency (JAXA) in Japan, and the K. A. Wallenberg Foundation, the Swedish Research Council and the Swedish National Space Board in Sweden.

Additional support for science analysis during the operations phase is gratefully acknowledged from the Istituto Nazionale di Astrofisica in Italy and the Centre National d'Études Spatiales in France.

Co-authors Briggs, Murphy, Schwartz, Share, and Tolbert were partially funded by the Fermi GI program to conduct the joint spectroscopic studies presented in this paper.

\section{REFERENCES}

Abdo, A. A., Ackermann, M., Ajello, M., et al. 2010, ApJ, 713, 154 Abdo, A. A., Ackermann, M., Ajello, M., et al. 2011, ApJ, 734, 116 Akimov, V. V., Ambrož, P., Belov, A. V., et al. 1996, Sol. Phys., 166, 107

Aschwanden, M. J. 2004, Physics of the Solar Corona. An Introduction (Chichester: Praxis)

Atwood, W. B., Abdo, A. A., Ackermann, M., et al. 2009, ApJ, 697, 1071 Bissaldi, E., von Kienlin, A., Lichti, G., et al. 2009, Exp. Astron., 24, 47

Briggs, M. S., Connaughton, V., Wilson-Hodge, C., et al. 2011, Geophys. Res. Lett., 38, L02808

Chupp, E. L., Forrest, D. J., Ryan, J. M., et al. 1982, ApJ, 263, L95

Chupp, E. L., \& Ryan, J. M. 2009, Res. Astron. Astrophys., 9, 11 
Cliver, E. W., Kahler, S. W., \& Vestrand, W. T. 1993, in 23rd Int. Cosmic Ray Conf., Vol. 3, ed. D. A. Leahy, R. B. Hickws, \& D. Venkatesan (Singapore: World Scientific), 91

Ellison, D. C., \& Ramaty, R. 1985, ApJ, 298, 400

Forrest, D. J., \& Chupp, E. L. 1983, Nature, 305, 291

Forrest, D. J., Vestrand, W. T., Chupp, E. L., Rieger, E., \& Cooper, J. 1986, Adv. Space Res., 6, 115

Forrest, D. J., Vestrand, W. T., Chupp, E. L., et al. 1985, in NASA. Goddard Space Flight Center 19th Int. Cosmic Ray Conf., Vol. 4, ed. M. GarciaMunoz, K. R. Pyle, \& J. A. Simpson, 146 (SEE N85-34729 23-92)

Hoover, A. S., Kippen, R. M., Wallace, M. S., et al. 2008, in AIP Conf. Ser. 1000, Gamma-ray Bursts 2007: Proc. Santa Fe Conf., ed. M. Galassi, D. Palmer, \& E. Fenimore (Melville, NY: AIP), 565

Kanbach, G., Bertsch, D. L., Fichtel, C. E., et al. 1993, A\&AS, 97, 349

Kane, S. R., Chupp, E. L., Forrest, D. J., Share, G. H., \& Rieger, E. 1986, ApJ, 300, L95

Kozlovsky, B., Lingenfelter, R. E., \& Ramaty, R. 1987, ApJ, 316, 801

Kozlovsky, B., Murphy, R. J., \& Share, G. H. 2004, ApJ, 604, 892

Kuznetsov, S. N., Kurt, V. G., Yushkov, B. Y., Kudela, K., \& Galkin, V. I. 2011, Sol. Phys., 268, 175

Martínez Oliveros, J. C., Couvidat, S., Schou, J., et al. 2011, Sol. Phys., 269, 269

McTiernan, J. M., \& Petrosian, V. 1990, ApJ, 359, 541

Meegan, C., Lichti, G., Bhat, P. N., et al. 2009, ApJ, 702, 791

Murphy, R. J., Dermer, C. D., \& Ramaty, R. 1987, ApJS, 63, 721

Murphy, R. J., Kozlovsky, B., Kiener, J., \& Share, G. H. 2009, ApJS, 183, 142

Murphy, R. J., Kozlovsky, B., Share, G. H., Hua, X., \& Lingenfelter, R. E. 2007, ApJS, 168, 167
Murphy, R. J., Share, G. H., Grove, J. E., et al. 1997, ApJ, 490, 883

Murphy, R. J., Share, G. H., Skibo, J. G., \& Kozlovsky, B. 2005, ApJS, 161, 495

Park, B. T., Petrosian, V., \& Schwartz, R. A. 1997, ApJ, 489, 358

Pelassa, V., Preece, R., Piron, F., et al. 2010, arXiv:1002.2617

Petrosian, V., McTiernan, J. M., \& Marschhauser, H. 1994, ApJ, 434, 747

Ramaty, R., Murphy, R. J., \& Dermer, C. D. 1987, ApJ, 316, L41

Rank, G., Ryan, J., Debrunner, H., McConnell, M., \& Schönfelder, V. 2001, A\&A, 378, 1046

Reames, D. V. 1995, Adv. Space Res., 15, 41

Rieger, E., \& Marschhäuser, H. 1991, in Max '91/SMM Solar Flares: Observations and Theory, ed. R. M. Winglee \& A. L. Kiplinger, 68

Ryan, J. M. 2000, Space Sci. Rev., 93, 581

Sakurai, T., Ichimoto, K., Hiei, E., et al. 1992, PASJ, 44, L7

Share, G. H., Chupp, E. L., Forrest, D. J., \& Rieger, E. 1983, in AIP Conf. Ser. 101, Positron-Electron Pairs in Astrophysics, ed. M. L. Burns, A. K. Harding, \& R. Ramaty (Melville, NY: AIP), 15

Share, G. H., Murphy, R. J., \& Ryan, J. 1997, in AIP Conf. Ser. 410, Proc. Fourth Compton Symp., ed. C. D. Dermer, M. S. Strickman, \& J. D. Kurfess (Melville, NY: AIP), 17

Shih, A. Y., Lin, R. P., \& Smith, D. M. 2009, ApJ, 698, L152

The Fermi-LAT Collaboration. 2011, arXiv:1108.1435v1

Thompson, D. J., Bertsch, D. L., Fichtel, C. E., et al. 1993, ApJS, 86, 629

Vestrand, W. T., Share, G. H., Murphy, R. J., et al. 1999, ApJS, 120, 409

Vilmer, N., MacKinnon, A. L., Trottet, G., \& Barat, C. 2003, A\&A, 412, 865

Young, C. A., Arndt, M. B., Bennett, K., et al. 2001, in AIP Conf. Ser. 587, Gamma 2001: Gamma-Ray Astrophysics, ed. S. Ritz, N. Gehrels, \& C. R. Shrader (Melville, NY: AIP), 613 


\section{ERRATUM: “FERMI DETECTION OF $\gamma$-RAY EMISSION FROM THE M2 SOFT X-RAY FLARE ON 2010 JUNE 12” (2012, ApJ, 745, 144)}

M. Ackermann ${ }^{1}$, M. Ajello ${ }^{2}$, A. Allafort ${ }^{2}$, W. B. Atwood ${ }^{3}$, L. Baldini ${ }^{4}$, G. Barbiellini ${ }^{5,6}$, D. Bastieri ${ }^{7,8}$, K. Bechtol ${ }^{2}$, R. Bellazzini ${ }^{4}$, P. N. Bhat ${ }^{9}$, R. D. Blandford ${ }^{2}$, E. Bonamente ${ }^{10,11}$, A. W. Borgland ${ }^{2}$, J. Bregeon ${ }^{4}$, M. S. Briggs ${ }^{9}$, M. Brigida ${ }^{12,13}$, P. Bruel ${ }^{14}$, R. Buehler ${ }^{2}$, J. M. Burgess ${ }^{9}$, S. Buson ${ }^{7,8}$, G. A. CAliandro ${ }^{15}$, R. A. CAMeron ${ }^{2}$, J. M. Casandian ${ }^{16}$, C. Cecchi ${ }^{10,11}$, E. Charles ${ }^{2}$, A. Chekhtman ${ }^{17,50}$, J. Chiang ${ }^{2}$, S. Ciprini ${ }^{11,18}$, R. Claus ${ }^{2}$, J. Cohen-Tanugi ${ }^{19}$, V. Connaughton ${ }^{9}$, J. Conrad ${ }^{20,21,51}$, S. Cutini ${ }^{22}$, B. R. Dennis ${ }^{23}$, F. De Palma ${ }^{12,13}$, C. D. Dermer ${ }^{24}$, S. W. Digel ${ }^{2}$, E. do Couto e Silva ${ }^{2}$, P. S. Drell ${ }^{2}$, A. Drlica-Wagner ${ }^{2}$, R. Dubois ${ }^{2}$, C. Favuzzi ${ }^{12,13}$, S. J. Fegan ${ }^{14}$, E. C. Ferrara $^{23}$, P. Fortin ${ }^{14}$, Y. Fukazawa ${ }^{25}$, P. Fusco ${ }^{12,13}$, F. Gargano ${ }^{13}$, S. Germani ${ }^{10,11}$, N. Giglietto ${ }^{12,13}$, F. Giordano ${ }^{12,13}$, M. Giroletti ${ }^{26}$, T. Glanzman ${ }^{2}$, G. Godfrey ${ }^{2}$, L. Grillo ${ }^{2}$, J. E. Grove ${ }^{24}$, D. Gruber ${ }^{27}$, S. Guiriec ${ }^{9}$,

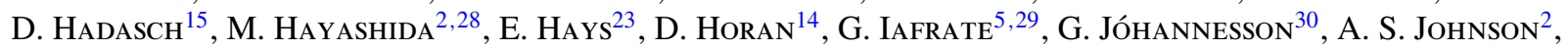

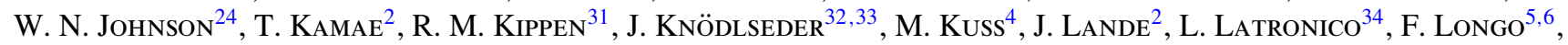

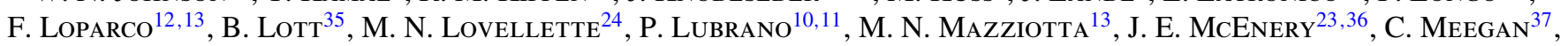
J. Mehault ${ }^{19}$, P. F. Michelson ${ }^{2}$, W. Mitthumsiri ${ }^{2}$, C. Monte $^{12,13}$, M. E. MonZani ${ }^{2}$, A. Morsellis ${ }^{38}$, I. V. Moskalenko², S. Murgia ${ }^{2}$, R. Murphy ${ }^{24}$, M. Naumann-Godo ${ }^{16}$, E. Nuss ${ }^{19}$, T. Nymark ${ }^{21,39}$, M. Ohno ${ }^{40}$, T. Ohsugi ${ }^{41}$, A. OKumura ${ }^{2,40}$, N. Omodei ${ }^{2}$, E. Orlando ${ }^{2,27}$, W. S. Paciesas ${ }^{9}$, J. H. Panetta ${ }^{2}$, D. Parent ${ }^{42,50}$, M. Pesce-Rollins ${ }^{4}$, V. Petrosian ${ }^{2}$,

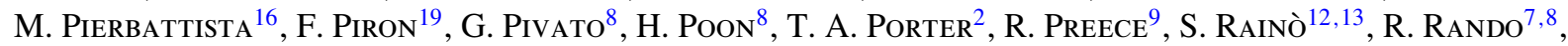
M. Razzano ${ }^{3,4}$, S. Razzaque ${ }^{42,50}$, A. Reimer ${ }^{2,43}$, O. Reimer ${ }^{2,43}$, S. Ritz ${ }^{3}$, C. Sbarra ${ }^{7}$, R. A. Schwartz ${ }^{23}$, C. Sgrò ${ }^{4}$,

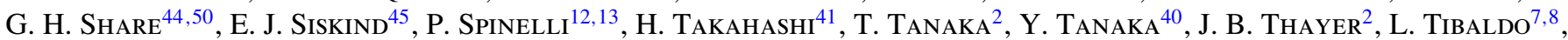
M. Tinivella ${ }^{4}$, A. K. TolberT ${ }^{23}$, G. Tosti ${ }^{10,11}$, E. Troja ${ }^{23,52}$, Y. UchiYama ${ }^{2}$, T. L. Usher ${ }^{2}$, J. VAndenbroucKe ${ }^{2}$, V. Vasileiou ${ }^{19}$, G. Vianello ${ }^{2,46}$, V. Vitale ${ }^{38,47}$, A. von Kienlin ${ }^{27}$, A. P. Waite ${ }^{2}$, C. Wilson-Hodge ${ }^{48}$, D. L. WooD ${ }^{49,50}$, K. S. Wood ${ }^{24}$, AND Z. YANG ${ }^{20,21}$

${ }^{1}$ Deutsches Elektronen Synchrotron DESY, D-15738 Zeuthen, Germany

${ }^{2}$ W. W. Hansen Experimental Physics Laboratory, Kavli Institute for Particle Astrophysics and Cosmology, Department of Physics and SLAC National Accelerator Laboratory, Stanford University, Stanford, CA 94305, USA; nicola.omodei@ gmail.com

${ }^{3}$ Santa Cruz Institute for Particle Physics, Department of Physics and Department of Astronomy and Astrophysics, University of California at Santa Cruz, Santa Cruz, CA 95064, USA ${ }^{4}$ Istituto Nazionale di Fisica Nucleare, Sezione di Pisa, I-56127 Pisa, Italy

${ }^{5}$ Istituto Nazionale di Fisica Nucleare, Sezione di Trieste, I-34127 Trieste, Italy; francesco.longo@ trieste.infn.it ${ }^{6}$ Dipartimento di Fisica, Università di Trieste, I-34127 Trieste, Italy

${ }^{7}$ Istituto Nazionale di Fisica Nucleare, Sezione di Padova, I-35131 Padova, Italy

${ }^{8}$ Dipartimento di Fisica "G. Galilei," Università di Padova, I-35131 Padova, Italy

${ }^{9}$ Center for Space Plasma and Aeronomic Research (CSPAR), University of Alabama in Huntsville, Huntsville, AL 35899, USA; michael.briggs@ nasa.gov

${ }^{10}$ Istituto Nazionale di Fisica Nucleare, Sezione di Perugia, I-06123 Perugia, Italy

${ }^{11}$ Dipartimento di Fisica, Università degli Studi di Perugia, I-06123 Perugia, Italy

${ }^{12}$ Dipartimento di Fisica “M. Merlin,” dell’Università e del Politecnico di Bari, I-70126 Bari, Italy

${ }^{13}$ Istituto Nazionale di Fisica Nucleare, Sezione di Bari, I-70126 Bari, Italy

${ }_{15}^{14}$ Laboratoire Leprince-Ringuet, École polytechnique, CNRS/IN2P3, Palaiseau, France

${ }^{15}$ Institut de Ciències de l'Espai (IEEE-CSIC), Campus UAB, E-08193 Barcelona, Spain

${ }^{16}$ Laboratoire AIM, CEA-IRFU/CNRS/Université Paris Diderot, Service d'Astrophysique, CEA Saclay, 91191 Gif sur Yvette, France

${ }^{17}$ Artep Inc., 2922 Excelsior Springs Court, Ellicott City, MD 21042, USA

${ }^{18}$ ASI Science Data Center, I-00044 Frascati (Roma), Italy

${ }^{19}$ Laboratoire Univers et Particules de Montpellier, Université Montpellier 2, CNRS/IN2P3, Montpellier, France

${ }^{20}$ Department of Physics, Stockholm University, AlbaNova, SE-106 91 Stockholm, Sweden

${ }^{21}$ The Oskar Klein Centre for Cosmoparticle Physics, AlbaNova, SE-106 91 Stockholm, Sweden

${ }^{22}$ Agenzia Spaziale Italiana (ASI) Science Data Center, I-00044 Frascati (Roma), Italy

${ }^{23}$ NASA Goddard Space Flight Center, Greenbelt, MD 20771, USA

${ }^{24}$ Space Science Division, Naval Research Laboratory, Washington, DC 20375-5352, USA

${ }^{25}$ Department of Physical Sciences, Hiroshima University, Higashi-Hiroshima, Hiroshima 739-8526, Japan ${ }^{26}$ INAF Istituto di Radioastronomia, I-40129 Bologna, Italy

${ }^{27}$ Max-Planck Institut für extraterrestrische Physik, D-85748 Garching, Germany; dgruber@ mpe.mpg.de

${ }^{28}$ Department of Astronomy, Graduate School of Science, Kyoto University, Sakyo-ku, Kyoto 606-8502, Japan

29 Osservatorio Astronomico di Trieste, Istituto Nazionale di Astrofisica, I-34143 Trieste, Italy

${ }^{30}$ Science Institute, University of Iceland, IS-107 Reykjavik, Iceland

${ }^{31}$ Los Alamos National Laboratory, Los Alamos, NM 87545, USA

${ }^{32}$ CNRS, IRAP, F-31028 Toulouse Cedex 4, France

${ }^{33}$ GAHEC, Université de Toulouse, UPS-OMP, IRAP, Toulouse, France

${ }^{34}$ Istituto Nazionale di Fisica Nucleare, Sezioine di Torino, I-10125 Torino, Italy

${ }^{35}$ Centre d'Études Nucléaires de Bordeaux Gradignan, Université Bordeaux 1, CNRS/IN2p3, F-33175 Gradignan, France

${ }^{36}$ Department of Physics and Department of Astronomy, University of Maryland, College Park, MD 20742, USA

${ }^{37}$ Universities Space Research Association (USRA), Columbia, MD 21044, USA

${ }^{38}$ Istituto Nazionale di Fisica Nucleare, Sezione di Roma "Tor Vergata," I-00133 Roma, Italy

${ }^{39}$ Department of Physics, Royal Institute of Technology (KTH), AlbaNova, SE-106 91 Stockholm, Sweden

${ }^{40}$ Institute of Space and Astronautical Science, JAXA, 3-1-1 Yoshinodai, Chuo-ku, Sagamihara, Kanagawa 252-5210, Japan

${ }^{41}$ Hiroshima Astrophysical Science Center, Hiroshima University, Higashi-Hiroshima, Hiroshima 739-8526, Japan 


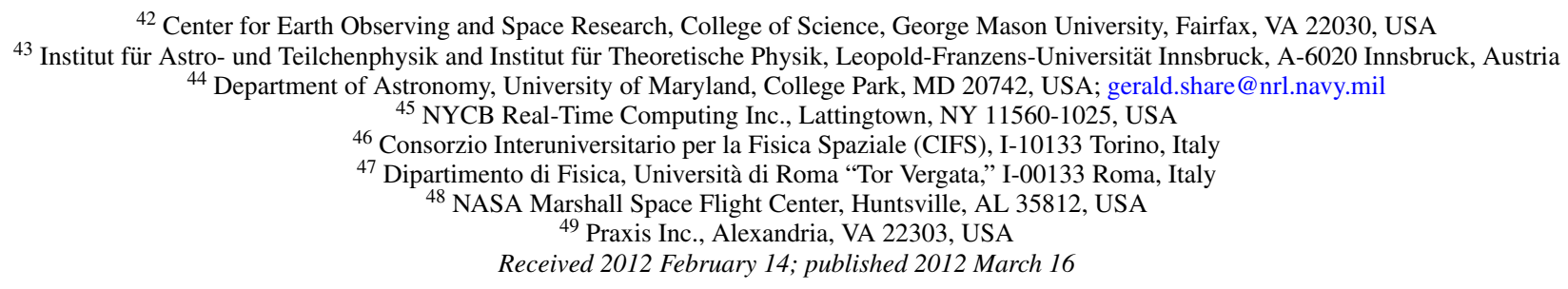

\section{ABSTRACT}

Due to an error at the publisher, the times given for the major tick marks in the $X$-axis in Figure 1 of the published article are incorrect. The correctly labeled times should be “00:52:00," "00:54:00,” . ., and "01:04:00.” The correct version of Figure 1 and its caption is shown below. IOP Publishing sincerely regrets this error.

Key words: acceleration of particles - Sun: flares - Sun: particle emission - Sun: X-rays, gamma rays

Online-only material: color figure

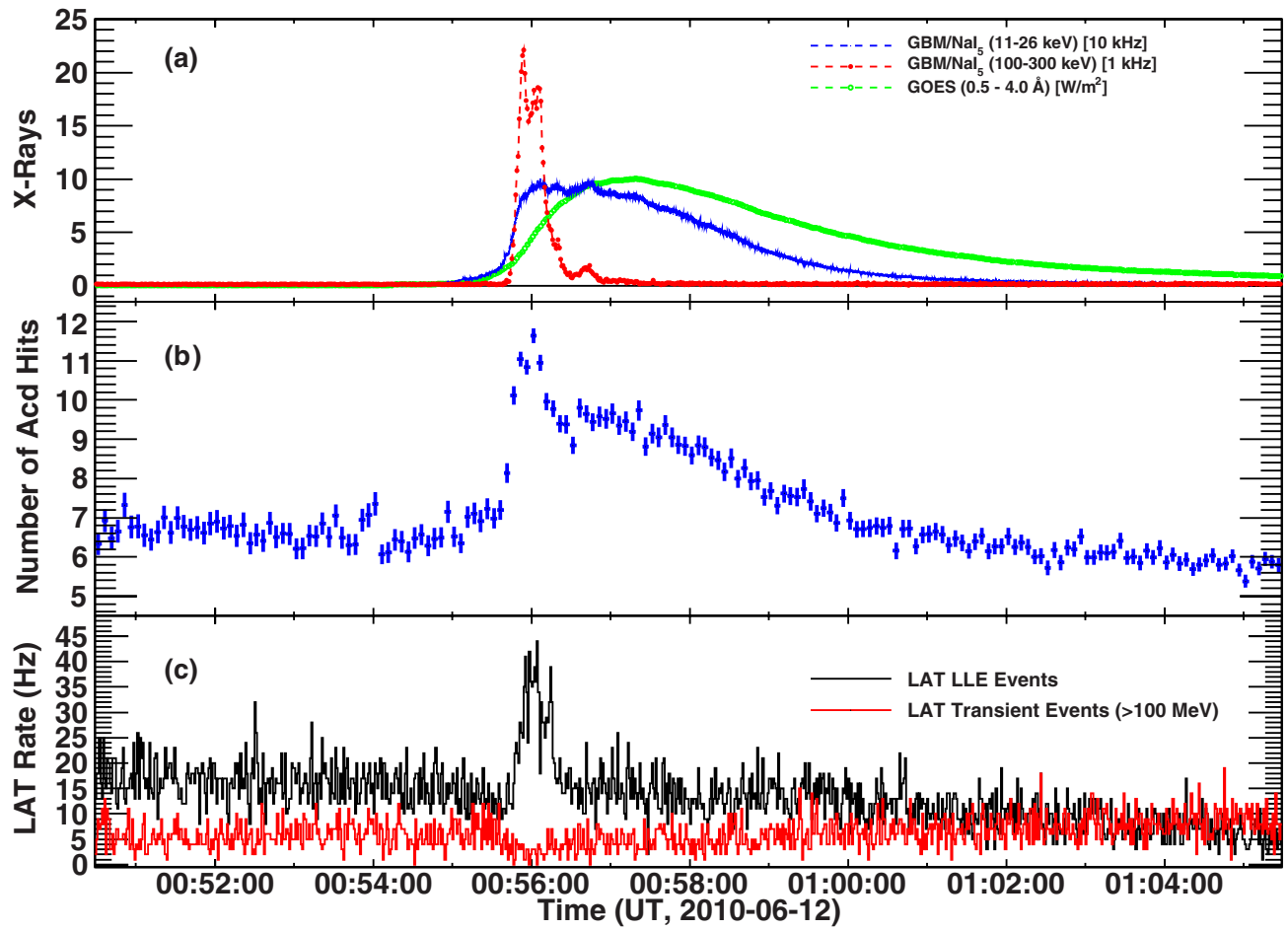

Figure 1. Time histories related to the 2010 June 12 solar flare. (a) GOES $0.5-4 \AA$ rates, and GBM Na I 11-26 keV and 100-300 keV relative rates; (b) LAT ACD hit rate $>100 \mathrm{keV}$ containing contributions from background, $>100 \mathrm{keV}$ solar flare X-rays (impulsive peak) and pulse pile-up from tens of keV solar X-rays following the Na I 11-26 keV profile in (a); and (c) LLE and LAT Transient Class event rates.

(A color version of this figure is available in the online journal.)

\footnotetext{
${ }^{50}$ Resident at Naval Research Laboratory, Washington, DC 20375, USA.

${ }^{51}$ Royal Swedish Academy of Sciences Research Fellow, funded by a grant from the K. A. Wallenberg Foundation.

52 NASA Postdoctoral Program Fellow, USA.
} 